\title{
ON THE POINT AND BLOCK FACTORIZATION ITERATIVE METHODS FOR ARBITRARY MATRICES AND THE CHARACTERIZATION OF M-MATRICES
}

R. Beauwens

Northwestern University
Evanston, Illinois

January 1974

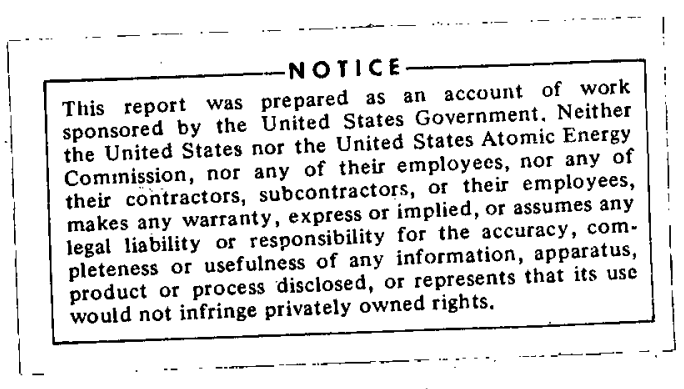

PREPARED FOR THE U. S. ATOMIC ENERGY COMMISSION UNDER CONTRACT NO. AT $(11-1)-2280$ 


\section{DISCLAIMER}

This report was prepared as an account of work sponsored by an agency of the United States Government. Neither the United States Government nor any agency Thereof, nor any of their employees, makes any warranty, express or implied, or assumes any legal liability or responsibility for the accuracy, completeness, or usefulness of any information, apparatus, product, or process disclosed, or represents that its use would not infringe privately owned rights. Reference herein to any specific commercial product, process, or service by trade name, trademark, manufacturer, or otherwise does not necessarily constitute or imply its endorsement, recommendation, or favoring by the United States Government or any agency thereof. The views and opinions of authors expressed herein do not necessarily state or reflect those of the United States Government or any agency thereof. 


\section{DISCLAIMER}

Portions of this document may be illegible in electronic image products. Images are produced from the best available original document. 
ON THE POINT AND CLOCKK FACTORIZATION ITERATIVE METHODS

FOR ARBITRARY MATRICES AND THE CHARACTERIZATION

OF M-MATRICES

by

R. Beauwens

\section{Abstract}

A new formulation of the point Buleev method is proposed. Factorizability conditions and convergence criteria of composite methods including the general 01iphant-Buleev-Woznicki method are obtained for arbitrary matrices. The generalization of these results to the block Oliphant-Buleev-Woznicki method is performed and a further generalization of these results to the new class of block-M-matrices is presented.

As auxiliary results, the refinement of previous convergence criteria for essentially non-positive matrices leads to a new formulation of some classical characterization properties of M-matrices, due to Fiedler and Ptak, and these properties are also generalized to partitioned M-matrices and block-M-matrices.

Finally, using an extension of a theorem of Woznicki, strict comparisons between the asymptotic convergence rates of some factorization iterative methods are obtained.

\footnotetext{
* Work supported by a NATO fellowship and in part by Brussels University, Northwestern University and the U.S. Atomic Energy Commission under Contract No. AT $(11-1)-2280$.
} 


\section{Introduction}

Block factorization iterative methods have been introduced recently [1]; however, the picture was not complete because the Buleev method and composite methods including the Buleev scheme could not be generalized. We show in the present report that it is possible to perform this gentralization with the help of a new formulation of the point Buleev method.

It turns out that the new formulation of the point Buleev method has several other advantages: it simplifies the proofs of factorizability conditions and convergence criteria considered in [1] and it makes possible to establish convergence criteria for arbitrary matrices, a problem which was left unsolved for schemes including the Buleev method, even in the case of diagonally dominant matrices [2].

Some auxiliary results will be established which give refinements of the factorizability conditions obtained in [1]; as a consequence, a new form of classical characterization properties of M-matrices, due to Fiedler and Pták [3], is obtained.

Finally, a new class of matrices will be introduced, the class of block-M-matrices and the extension of our analysis of block methods to this class of matrices will be indicated.

Al1 notations used in the subsequent sections are taken from section 2 of ref. [1] and their definitions will not be reproduced here. Therefore we shal1 also keep the terminology defined in [1]; we learned however recently, and after completion of most parts of the present work, that the Woznicki methods have first been considered by Varga [14] who, moreover, provided a convergence analysis for stieltjes matrices. It would thus be more appropriate to call these methods the Varga or Varga-Woznicki methods. 
2. Point methods

\subsection{The point Buleey niethod}

The point Buleev method has been defined in ref. [1] by

$$
\begin{aligned}
& E_{1}=E \quad F_{1}=F \\
& P=(I+\alpha) D-\operatorname{diag}\left(E^{-1} F S\right)
\end{aligned}
$$

where $\alpha$ is a diagonal matrix of arbitrary parameters, and $S$ is the matrix whose entries are a11 equal to 1 . Other notations are defined in section 2 of [1]. This definition is a generalization of the method used by Dupont Kenda11 and Rachford [4] for the multidimensional diffusion equation and the equivalence between this method and the method first introduced by Buleev [5] has been shown in [6].

If we define the diagonal matrices ' $\alpha^{\prime}$ and $\beta$ by

$$
\begin{aligned}
& \alpha^{\prime}=\left(\operatorname{diag}\left(\operatorname{EP}^{-1} F S\right)-\operatorname{diag}\left(E P^{-1} F\right)\right) D^{-1} \\
& \beta=I+\alpha-\alpha^{\prime} .
\end{aligned}
$$

We obtain from Eq.(2),

$$
P=\beta D-\operatorname{diag}\left(E P^{-1} F\right) \text {. }
$$

Therefore, an equivalent definition of the point Buleev method may be written

$$
\begin{array}{lc}
E_{1}=E & F_{1}=F \\
P=\beta D & -\operatorname{diag}\left(E P^{-1} F\right)
\end{array}
$$

where $\beta=\left(\beta_{i i}\right)$ is a diagonal matrix of given iteration parameters.

We shall henceforth use this latter definition. The corresponding splitting is given by

$$
\begin{aligned}
& M=(P-E) P^{-1}(P-F) \\
& N=(B-I) D+\operatorname{offdiag}\left(E P^{-1} F\right) .
\end{aligned}
$$

We have intentionally used the notation $\beta$ in conjunction with the similar notation introduced in [1] for the oliphant method. No confusion 
can arise since, here, $\beta$ is defined only for the diagonal entries while for the oliphant method, $\beta$ is derined only for the off-diagonal entries. In this way, the Oliphant and Buleev techniques appear as complementary relaxation procedures applied to the EWA method of Woznicki.

\subsection{Composite methods}

The definitions of the composite methods of Oliphant-Buleev, Buleev-Woznicki and Oliphant-Buleev-Woznicki are accordingly modified. We shall simply quote here the new definitions for reference purposes, using the notations of $[1]$.

The 0liphant-Buleev method

$$
\begin{aligned}
& E_{1}=E_{\beta} \quad F_{1}=F_{\beta} \\
& P=D_{\beta}-\operatorname{diag}\left(E_{\beta} P^{-1} F_{\beta}\right) \\
& D_{\beta}=\left(\beta_{i i} a_{i i}\right) \quad i \in[1, n] \\
& E_{\beta}=-\left(\beta_{i s} a_{i s}\right) \quad(i, s) \in V_{E} \\
& F_{\beta}=-\left(\beta_{s j} a_{s j}\right) \quad(s, j) \in V_{F} \\
& M=\left(P-E_{\beta}\right) P^{-1}\left(P-F_{\beta}\right) \\
& N=\left(D_{\beta}-D\right)+\left(E-E_{\beta}\right)+\left(F-F_{\beta}\right)+\operatorname{offdiag}\left(E_{\beta} P^{-I_{F}}\right)
\end{aligned}
$$

The Buleev-Woznicki method

$$
\begin{aligned}
& E_{1}=E+G \quad F_{1}=F+H \\
& P=D_{\beta}-\operatorname{diag}\left((E+G) P^{-1}(F+H)\right) \\
& G+H=\operatorname{rest}_{W}\left((E+G) P^{-1}(F+H)\right) \\
& D_{B}=\left(\beta_{i i}{ }_{i i}\right) \quad i \ddot{e}[1, n] \\
& M=(P-E-G) P^{-1}(P-F-H) \cdot \\
& N=\left(D_{\beta}-D\right)+\left(\operatorname{offdiag}\left((E+G) P^{-1}(F+H)\right)-(G+H)\right)
\end{aligned}
$$


The Oliphant-Buleev-Woznicki method

$$
\begin{aligned}
& E_{1}=E_{B}+G \quad F_{1}=F_{\beta}+H \\
& P=D_{\beta}-\operatorname{diag}\left(\left(E_{\beta}+G\right) P^{-1}\left(F_{\beta}+H\right)\right) \\
& G+H=\operatorname{rest}_{W}\left(\left(E_{B}+G\right) P^{-1}\left(F_{\beta}+H\right)\right) \\
& D_{\beta}=\left(\beta_{i i}{ }_{i i}\right) \quad i \in[1, n] \\
& E_{\beta}=-\left(\beta_{i s}{ }^{a}{ }_{i s}\right) \quad(i, s) \in V_{E} \\
& F_{\beta}=-\left(\beta_{s j^{2} s j}\right) \quad(s, j) \varepsilon^{V_{F}} \\
& M=\left(P-E_{\beta}-G\right) P^{-1}\left(P-F_{\beta}-H\right) \\
& N=\left(D_{\beta}-D\right)+\left(E-E_{\beta}\right)+\left(F-F_{\beta}\right) \\
& +\left(\operatorname{offdiag}\left(\left(E_{\beta}+G\right) \mathrm{P}^{-1}\left(\mathrm{~F}_{\beta}+\mathrm{H}\right)\right)-(G+H)\right)
\end{aligned}
$$


3. Factorizability conditions and convergence criteria for point methods

\subsection{Essentially non-positive matrices}

Essentialiy non-positive matrices have been considered in [1] where sufficient criteria have been obtained. Necessary conditions will be considered here. For the sufficient criteria, it may be observed that the proufs given in [1] are much simplified by the use of the new definition of the Buleev method, and that the analysis presented there for composite methods including the Buleev scheme can be based on lema 1 and lemma 3 of [1]. For comparisons with the new definition, it must be noted that the condition $\alpha \geq \alpha^{0}$ used in [1] has now to be replaced by $\beta_{i i} \geq 1$ for $i \in[1, n]$.

For the necessary conditions, some of our proofs will be based on a strengthened form of theorem 3.13 of Varga [7], first given by Ortega and Rheinboldt [8]. A similar theorem, slightly more general, was published a year later by Price [9]. A still more general formulation has been given by Vandergraft $[10]$.

Summarizing the results which will be needed here we introduce

\section{Definition 1}

For $n \times n$ real matrices $A, M$ and $N, A=M-N$ is a weak regular splitting of $A$ if $M$ is non-singular with $M^{-1} \geq 0$ and (at least) $M^{-1} N \geq 0$ or $\mathrm{NM}^{-1} \geq 0$

Then we have $[8],[9],[10]$ :

\section{Theorem 1}

Let $A=M-N$ be a weak regular splitting of the (rea1) matrix $A$ and $T=M^{-1} N$ be the associated iteration matrix. Then $\rho(T)<1$ if and only if $\mathrm{A}$ is monotone.

\subsection{The Oliphant method}

According to Varga [7], an essentially non-positive matrix is a 
real in $x \mathrm{n}$ matrix whose off-diagonal elements are non-positive.

A general criterion which insures that the diagonal matrix $P$ of the Oliphant method is positive ${ }^{1}$. has been obtained in [1] for this class of matrices: if $A$ is an $M$-matrix and $0 \leq \beta_{i j} \leq 1$ for $(i, j) \in V$, then $P>0$.

The same condition is not necessary as shown by the following example

$$
A=\left[\begin{array}{ccc}
1 & -1 & -1 \\
-1 & 2 & -2 \\
-1 & -2 & 6
\end{array}\right] \quad B=\left[\begin{array}{lll}
0 & 1 & 1 \\
1 & 0 & 1 \\
1 & 1 & 0
\end{array}\right] \quad P=\left[\begin{array}{lll}
1 & 0 & 0 \\
0 & 1 & 0 \\
0 & 0 & 1
\end{array}\right]
$$

It is, however, a necessary condition for convergence and we have

\section{Theorem 2}

Let $A$ be essentialily non-positive and $0 \leq \beta_{i j} \leq 1$ for $(i, j) \in V$; then $\mathrm{P}>0$ and the Oliphant method converges if and only if $\mathrm{A}$ is an M-matrix.

\section{$\underline{\text { Proof }}$}

For the sufficient condition, we refer to $[1]$; for the necessary condition, assume that $\mathrm{P}>0$; then

$$
\begin{aligned}
& M=P\left(I-P^{-1} E_{\beta}\right)\left(I-P^{-1} F_{\beta}\right) \\
& M^{-1}=\left(I-P^{-1} F_{\beta}\right)^{-1}\left(I-P^{-1} E_{\beta}\right)^{-1} P^{-1}
\end{aligned}
$$

and

$$
N=\left(E-E_{\beta}\right)+\left(F-F_{\beta}\right)+\operatorname{offdiag}\left(E_{\beta} P^{-1} F_{\beta}\right)
$$

Thus, it is seen that $M^{-1} \geq 0$ and $N \geq 0$ when $P>0$ and $0 \leq \beta_{i j} \leq I$ for $(i, j) \in V$; in other words, the splitting $A=M-N$ is a regular splitting and the conclusion follows from theorem 1 .

\subsection{Composite methods}

The same method of proof applies to composite methods and we have

\footnotetext{
We use here the convention of ref. [1]: for diagonal matrices, $P>0$ means that $p_{i i}>0$ for $i=1,2 \ldots n$.
} 
the following result which completes theoren 6 of $[1]$.

Theorem 3

Let $A$ be essertially non-positive and $0 \leq B_{i j} \leq 1$ for $(i, j) \in V$ while $B_{i i} \geq 1$ for $i \in[1, n]$; then $P>0$ and the 01iphant-Buleev-Woznicki method converges if and only if $A$ is an M-matrix.

\subsection{The Choleski factorization and the characterization of M-matrices}

\subsection{The Choleski factorization of M-matrices}

As stated above, the positivity of $\mathrm{P}$ is generally not a necessary condition for the monotonicity of A. There exists, however, an exception, when the Woznicki method reduces to the Choleski factorization. More precisely we have:

Lemma 1

Let $\mathrm{A}=\mathrm{K}-\mathrm{L}-\mathrm{U}$ where $\mathrm{K} \geq 0, \mathrm{~L} \geq 0$ and $\mathrm{U} \geq 0$ are respectively diagonal, strictly lower and upper triangular matrices and let the diagonal, strictly lower and upper triangular matrices $P, G$, and $H$ respectively be defined by

$$
\begin{aligned}
& P=K-\operatorname{diag}\left((L+G) P^{-1}(U+H)\right) \\
& G+H=\operatorname{offdiag}\left((L+G) P^{-1}(U+H)\right) .
\end{aligned}
$$

Then $P>0$ if and only if $A$ is an M-matrix.

Proof

For the sufficient condition, we refer to [1]; for the necessary condition, assume that $P>0$; then, from (35) and (36), we have

$$
\begin{aligned}
& G \geq 0 \text { and } H \gtrless 0 \\
& A=K-L-U=(P-L-G) P^{-1}(P-U-H)
\end{aligned}
$$

and

$$
A^{-1}=(P-U-H)^{-1} P(P-L-G)^{-1} \text {. }
$$

But

$$
\begin{aligned}
& (P-U-H)^{-1}=P \sum_{k=0}^{n-1}\left(P^{-1}(U+H)\right)^{k} \\
& (P-L-G)^{-1}=P \sum_{k=0}^{n-1}\left(P^{-1}(L+G)\right)^{k}
\end{aligned}
$$


and, therefore, $A^{-1} \geq 0$, which completes the proof.

An auxiliary consequence of this result is the possibility of

establishing ipper bounds for the leading principal minors of A:

\section{Theorem 4}

Let $A=K-I-U$ where $K \sum 0, L \geq 0$ and $U \geq 0$ are respectively diagonal, strictly lower and upper triangular matrices be an M-matrix and let $D_{i}$ be its leading principal minors; then

$$
D_{i} \leq \prod_{s=1}^{i} p_{\text {ss }}
$$

where $\mathrm{P}_{S S}$ are the entries of a diagonal matrix $\mathrm{P}$ defined by

$$
\begin{aligned}
& P=K-\operatorname{diag}\left((L+G) P^{-1}(U+H)\right) \\
& G+H=\operatorname{rest}_{W}\left((L+G) P^{-1}(U+H)\right)
\end{aligned}
$$

$G$ and $H$ are respectively strictly lower and upper triangular and $W$ is an arbitrary set of off-diagonal indices. Moreover if $W$ contains the Choleski set of $A$, then

$$
D_{i}=\prod_{s=1}^{i} p_{s s}
$$

$\underline{\text { Proof }}$

Let $A_{i}, P_{i}, L_{i}, U_{i}, G_{i}$ and $H_{i}$ be the leading principal submatrices of $A, P, L, U, G$ and $H$; if $W$ is the complete set of off-diagonal indices, we have from $\mathrm{Eq} \cdot(37)$

$$
D_{i}=\operatorname{det}\left(A_{i}\right)=\operatorname{det}\left(P_{i}-L_{i}-G_{i}\right) \operatorname{det}\left(P_{i}^{-1}\right) \operatorname{det}\left(P_{i}-U_{i}-H_{i}\right)=\prod_{s=1}^{i} p_{s s}
$$

From lemma 3 of $[1]$, we know that $P$ is a non-increasing function of the set $W$; therefore

$$
D_{i} \leqslant \prod_{s=1}^{i} p_{s s}
$$

with equality when $W$ is greater or equal to the Choleski set of $A$. 
Corollary

Let $A$ and $A^{\prime}$ be essentially non-negative matrices such that $A \leq A$, and let $D_{i}$ and $D_{i}^{\prime}$ be their leading principal minors.

If $A$ is monocone, then $A^{\prime}$ is monotone and

$$
D_{i} \leq D_{i}^{\prime} \quad \ldots i=1,2 \ldots n
$$

$\underline{\text { Proof }}$

It foilows from theorem 1 of [1] that $A^{\prime}$ is monotone; on the other hand, we have

$$
D_{i}=\prod_{s=1}^{i} P_{s s} \quad \text { and } \quad D_{i}^{\prime}=\prod_{s=1}^{i} p_{s s}^{\prime}
$$

where $\mathrm{P}$ and $\mathrm{P}^{\prime}$ are defined by Eq. (35) and (36) applied to $\mathrm{A}$ and $\mathrm{A}^{\prime}$ and, from lemma 6 of [1],

$$
\mathrm{p}_{\mathrm{sS}} \leq \mathrm{p}_{\mathrm{sS}}^{\prime}, \quad \mathrm{s}=1,2 \ldots . \mathrm{n}
$$

which completes the proof.

\subsection{The characterization of M-matrices}

The subject of finding criteria which insure that an essentially non-positive matrix is an M-matrix, has been considered in much detail by Fiedler and Pták [3] and the criteria given by theorem 2 , theorem 3 and lemma 1 can be added to their list.

Actually a closer connection can be established and it is possible to give, on the basis of lemma 1 and theorem 4, new forms of two criteria first obtained by Fiedler and Pták.

Let $\mathrm{N}$ denote the set of indices 1,2 . . $\mathrm{n}$; consider matrices as real functions on $\mathrm{P} \times \mathrm{P}$ where $\mathrm{P}$ is some index set; if $\mathrm{M} \subset \mathrm{N}$ and if $A$ is a matrix on $N \times N$, then let $A(M)$ denote the partial function of $A$ on $M \times M$ : it is the principal submatrix of $A$ corresponding to $M$ and det $A(M)$ is the principal minor of $A$ corresponding to $M$. 
With these notations, we have from the work of Fiedler and Pták [3]: Theorem 5

Let $A$ be an essentially non-positive matrix. Then the following conditions are equivalent to each: other:

$1^{\circ} /$ there exists a vector $\mathrm{x} \geq 0$ such that $\mathrm{Ax}>0$;

$2 \%$ there exists a strictly increasing sequence $0 \neq M_{1} \subset M_{2} \subset \ldots \subset M_{n}=N$ such that the principal minors $\left.\operatorname{det} \mathrm{A}_{(1}\right)$ are positive;

$3^{\circ} /$ there exists a permutation matrix $P$ such that $\mathrm{PAP}^{-1}$ may be written in the form $R S$ where $R$ and $S$ are respectively lower and upper essentially non-positive triangular matrices with positive diagonal elements.

Using the same notations, we have

Theorem 6

Let $A$ be an essentially non-positive matrix. Then the following conditions are equivalent to each other:

$1^{\circ} /$ there exists a vector $\mathrm{x} \geq 0$ such that $\mathrm{Ax}>0$;

$2 \%$ the leading principal minors of A are positive;

$3 \%$ t the matrix A may be written in the form $R S$ where $R$ and $S$ are respectively lower and upper essentially nonmpositive triangular matrices with positive diagonal elements.

\section{$\underline{\text { Proof }}$}

Implication $1^{\circ}$ to $2^{\circ}$ follows from theorem 4 . Implication $3^{\circ}$ to $1^{\circ}$ was done in the proof of lemma 1 (and, actually, the lemma 1 states the equivalence between $1^{\circ}$ and $3^{\circ}$ ). It remains to prove the implication $2^{\circ}$ to $3^{\circ}$ and this is the subject of the proposition (3.1) of Fiedler and Pták [3].

Remark

By using the method of proof of Fiedler and Ptak, a direct proof of theorem 6 is obtajned thus providing an alternate and much simpler proof of lema 3 of $[1]$. 


\section{$3: 3$ Criteria for general matrices}

Let $A$ be an arbitrary complex matrix. Following Fiedler and Pták, we shall introduce the essentially nor-negative matrix $P(A)$ defined as

$$
P(A)=|K|-|L|-|U|
$$

where

$$
A=K-L-U
$$

is the decomposition of A into its diagonal, strictly lower and strictly upper triangular parts respectively and $|\mathrm{K}|,|\mathrm{L}|,|\mathrm{U}|$ denote the matrices whose entries are the moduli of the corresponding entries of $\mathrm{K}, \mathrm{L}, \mathrm{U}$ respectively.

Some of the following results generalize criteria first obtained by Beauwens and Borysievicz [2] for diagonally dominant matrices. The generalization is twofold: first, the condition of strict or irreducible diagonal dominance is replaced by the more general condition of monotonicity; secondly, composite methods including the Buleev scheme are covered.

As in ref. $[2]$, the proaf of the convergence theorem for general matrices is based on theorem 2.8 of Varga [7] and we quote. it here for reference purpose:

Theorem 7 (Varga)

Let $A$ and $B$ be two $n \times n$ matrices with $0 \leq|B| \leq A$. Then

$$
\rho(B) \leq \rho(A)
$$

\section{Lemma 2}

Let $\mathrm{A}=\mathrm{K}-\mathrm{L}-\mathrm{U}$ where $\mathrm{K}, \mathrm{L}$ and $\mathrm{U}$ are respectively diagonal, strictly lower and upper triangular matrices, be an arbitrary complex matrix and let the diagonal, strictly lower and upper triangular matrices $P, G$ and $H$ respectively be defined by 


$$
\begin{aligned}
& P=K-\operatorname{diag}\left((L+G) P^{-1}(U+H)\right) \\
& G+H=\operatorname{rest}_{W}\left((L+G) P^{-1}(U+H)\right)
\end{aligned}
$$

where $W$ is an arbitrary set of offdiagonal entries, and let $\mathrm{P}^{\prime}, \mathrm{G}^{\prime}$ and $H^{\prime}$ be defined by the same relations applied to $|\mathrm{K}|,|\mathrm{L}|$ and $|\mathrm{U}|$.

If $P(A)$ is an M-natrix, then $P$ is non-singular; we have moreover

$$
|P| \geq P^{\prime}>0 \quad|G| \leq G^{\prime} \quad|H| \leq H^{\prime}
$$

\section{Proof}

Let $\mathrm{p}_{i i}, \mathrm{k}_{i \mathrm{i}}, \mathrm{l}_{i s}, \mathrm{~g}_{i s}, \mathrm{u}_{\mathrm{s} j}$ and $\mathrm{h}_{\mathrm{s} j}$ denote the entries of $\mathrm{P}, \mathrm{k}$, $L, G, U$ and $H$ respectively and let $p_{i i}^{\prime}, k_{i i}^{\prime}, 1_{i s}^{\prime}, g_{i s}^{\prime}, u_{s j}^{\prime}$ and $h_{s j}^{\prime}$ denote the entries of $P^{\prime},|K|,|L|, G^{\prime},|U|$ and $H^{\prime}$ respectively.

From Eq. (53) and (54) we have

$$
\begin{aligned}
& p_{i i}=k_{i i}-\sum_{s=1}^{i-1} \frac{\left(1_{i s}+g_{i s}\right)\left(u_{s i}+h_{s i}\right)}{p_{s s}} \\
& \sum_{s=1}^{i-1} \frac{\left(1_{k s}+g_{k s}\right)\left(u_{s i}+h_{s i}\right)}{p_{s s}} \quad \text { if }(k, i) \in W \\
& g_{k i}= \begin{cases}0 & \text { if }(k, i) \notin W\end{cases} \\
& h_{i k}= \begin{cases}\sum_{s=1}^{i-1} \frac{\left(1_{i s}+g_{i s}\right)\left(u_{s k}+h_{s k}\right)}{P_{s s}} & \text { if }(i, k) \in W>i \\
0 & \text { if }(i, k) \in W\end{cases}
\end{aligned}
$$

and the same relations hold between $p_{s s^{\prime}}^{\prime}, k_{i i^{\prime}}^{\prime} 1_{i s^{\prime}}^{\prime} g_{i s}^{\prime}, u_{s j}^{\prime}$ and $h_{s j}^{\prime}$

We prove by induction that

$$
\left|p_{s s}\right| \geq p_{s s}^{\prime}
$$




$$
\left|g_{k s}\right| \leq g_{k s}^{\prime} \quad \text { and } \quad\left|h_{s k}\right| \leq h_{s k}^{\prime} \quad \text { for } k>s
$$

For $s=1$, these relations are evident. Assume that they hold for $s \leq i-1$ and consider $k>i$. If $(k, i) \notin . W$, we have

$$
\left|g_{k i}\right|=g_{k i}^{\prime}=0
$$

while if $(k, j) \in W$

$$
\left|g_{k i}\right| \leq \sum_{s=1}^{i-1}\left|\frac{\left(1_{k s}+g_{k s}\right)\left(u_{s i}+h_{s i}\right)}{p_{s s}}\right| \leq \sum_{s=1}^{i-1} \frac{\left(1_{k s}^{\prime}+\left|g_{k s}\right|\right)\left(u_{s i}^{\prime}+\left|h_{s i}\right|\right)}{\left|p_{s s}\right|}
$$

and, therefore, by the induction hypothesis

$$
\left|g_{k i}\right| \leq \sum_{s=1}^{i-1} \frac{\left(1_{k s}^{\prime}+g_{k s}^{\prime}\right)\left(u_{s i}^{\prime}+h_{s i}^{\prime}\right)}{p_{s s}^{\prime}}=g_{k i}^{\prime}
$$

Similarly, we have

$$
\left|h_{i k}\right| \leq h_{i k}^{\prime}
$$

for $k>i$

For $\mathrm{p}_{i i}$, we have from $\mathrm{Eq} .(56)$

$$
\begin{gathered}
\left|p_{i i}\right| \geq\left|k_{i i}\right|-\left|\sum_{s=1}^{i-1} \frac{\left(1_{i s}+g_{i s}\right)\left(u_{s i}+k_{s i}\right)}{p_{s s}}\right| \geq k_{i i}^{\prime}-\sum_{s=1}^{i-1}\left|\frac{\left(1_{i s}+g_{i s}\right)\left(u_{s i}+h_{s i}\right)}{p_{s s}}\right| \\
\geq k_{i i}^{\prime}-\sum_{s=1}^{i-1} \frac{\left(1_{i s}^{\prime}+\left|g_{i s}\right|\right)\left(u_{s i}+\left|h_{s i}\right|\right)}{\left|p_{s s}\right|}
\end{gathered}
$$

and, by the induction hypothesis

$$
\left|p_{i i}\right| \geq k_{i i}^{\prime}-\sum_{s=1}^{i-1} \frac{\left(1_{i s}^{\prime}+g_{i s}^{\prime}\right)\left(u_{s i}^{\prime}+k_{s i}^{\prime}\right)}{p_{s s}^{\prime}}=p_{i i}^{\prime}
$$

The conclusion follows from lenma 3 of [1].

\section{Corollary}

Let $\mathrm{A}$ be an ärbitrary complex matrix; if $\mathrm{P}(\mathrm{A})$ is an M-matrix, then 
$A$ is non-singular and

$$
\left|A^{-1}\right| \leq(P(A))^{-1}
$$

$\underline{\text { Proof }}$

Let the diagonal, strictly lower and upper triangular matrices

$P, G$ and $H$ respectively be defined by

$$
\begin{aligned}
& P=K-\operatorname{diag}\left((I+G) P^{-1}(U+H)\right) \\
& G+H=\operatorname{offdiag}\left((L+G) P^{-1}(U+H)\right)
\end{aligned}
$$

and let $\mathrm{P}^{\prime}, \mathrm{G}^{\prime}$ and $\mathrm{H}^{\prime}$ be defined by the same relation applied to $|\mathrm{K}|$, $|\mathrm{L}|$ and $|\mathrm{U}|$.

From lemma 2, $\mathrm{P}$ is not singular so that these relations are meaninfgul. From lemma 3 of $[1]$, the same conclusion holds for $P$ '.

Thus we have successively

$$
\begin{aligned}
& A=(P-L-G) P^{-1}(P-U-H) \\
& A^{-1}=\left(I-P^{-1}(U+H)\right)^{-1}\left(I-P^{-1}(L+G)\right)^{-1} P^{-1} \\
& A^{-1}=\left(\sum_{k=0}^{n-1}\left(P^{-1}(U+H)\right)^{k}\right)\left(\sum_{I=0}^{n-1}\left(P^{-1}(L+G)\right)^{1}\right) P^{-1}
\end{aligned}
$$

and, therefore

$$
\left|A^{-1}\right| \leq\left|\sum_{k=0}^{n-1}\left(P^{-1}(U+H)\right)^{k}\right| \cdot\left|\sum_{1=0}^{n-1}\left(P^{-1}(L+G)\right)^{1}\right| \cdot\left|P^{-1}\right|
$$

thus also

$$
\left|A^{-1}\right| \leq\left[\sum_{k=0}^{n-1}\left(\left|P^{-1}\right|(|U|+|H|)\right)^{k}\right]\left[\sum_{1=0}^{n-1}\left(\left|P^{-1}\right|(|L|+|G|)\right)^{1}\right]\left|P^{-1}\right|
$$

and by lemma 2

$$
\left|A^{-1}\right| \leq\left[\sum_{k=0}^{n-1}\left(P^{-1}\left(|U|+H^{-}\right)\right)^{k}\right]\left[\sum_{1=0}^{n-1}\left(P^{-1}\left(|L|+G^{\prime}\right)\right)^{1}\right] P^{-1}=(P(A))^{-1}
$$

where the notations of 1 emma 2 have been used 


\section{Theorem 8}

If $P(A)$ is an M-matrix, $0 \leq \beta_{i j} \leq 1$ for $(i, j) \in V$ and $\beta_{i i} \geq 1$ for $i \in[l, n]$, then the Oliphant-Buleev-Woznicki method is convergent. hiorewver its asymptotic convergence rate is not less than the asymptotic comergence rate of the same method applied to $P(A)$.

Eroof

Let $A=M-N$ and $P(A)=M^{\prime}-N^{\prime}$ be the splittings of $A$ and $P(A)$. relative to the Oliphant-Buleev-Woznicki scheme.

We have successive1y

$$
\begin{gathered}
M=P\left(I-P^{-1}\left(E_{\beta}+F\right)\right)\left(I-P^{-1}\left(F_{B}+H\right)\right) \\
M^{-1}=\left(I-P^{-1}\left(F_{\beta}+H\right)\right)^{-1}\left(I-P^{-1}\left(E_{B}+G\right)\right)^{-1} P^{-1} \\
\left.M^{-1}=\sum_{k=0}^{n-1}\left(P^{-1}\left(F_{B}+H\right)\right)^{k}\right]\left[\sum_{1=0}^{n-1}\left(P^{-1}\left(E_{B}+G\right)\right)^{1}\right] P^{-1}
\end{gathered}
$$

thus

$$
\left|M^{-1}\right| \leq\left[\sum_{k=0}^{n-1}\left(\left|P^{-1}\right|\left(\left|F_{\beta}\right|+|H|\right)\right)^{k}\right]\left[\sum_{1=0}^{n-1}\left(\left|P^{-1}\right|\left(\left|E_{\beta}\right|+|G|\right)\right)^{1}\right]\left|P^{-1}\right|
$$

- and, therefore, by: lemma 2

$$
\left|M^{-1}\right| \leq\left[\sum_{k=0}^{n-1}\left(P^{-1}\left(F_{\beta}^{\prime}+H^{\prime}\right)\right)^{k}\right]\left[\sum_{1=0}^{n-1}\left(P^{-1}\left(E_{\beta}^{\prime}+G^{\prime}\right)\right)^{1}\right] P^{-1}=M^{-1}
$$

where the following notations are used

$$
\begin{aligned}
& E_{\beta}^{\prime}=|E|_{\beta} \\
& F_{\beta}^{\prime}=|F|_{\beta}
\end{aligned}
$$

and $P^{\prime}, G^{\prime}$ and $H^{\prime}$ are the matrices $P, G$ and $H$ of the Oliphant-BuleevWoznicki method applied to $P(A)$.

On the other hand, 


$$
\begin{aligned}
& \left.N=\left(D_{\beta}-D\right)+\left(E-E_{\beta}\right)+\left(E-F_{\beta}\right)+\operatorname{offdiag}\left(\left(E_{\beta}+G\right) P^{-1}\left(F_{\beta}+H\right)\right)-(G+H)\right) \\
& |N| \leq\left|D_{\beta}-D\right|+\left|E-E_{\beta}\right|+\left|F-F_{\beta}\right|+\left|\operatorname{offdiag}\left(\left(E_{\beta}+G\right) P^{-1}\left(F_{\beta}+H\right)\right)-(G+H)\right|
\end{aligned}
$$

But, since $\beta_{i i} \geq 1$ and $0 \leq \beta_{i j}<1$ for $(i, j) \in V$,

$$
\begin{aligned}
& \left|D_{\beta}-D\right|=|D|_{\beta}-|D|=D_{\beta}^{\prime}-D^{\prime} \\
& \left|E-E_{\beta}\right|=|E|-|E|_{\beta}=E^{\prime}-E_{\beta}^{\prime} \\
& \left|F-F_{\beta}\right|=|F|-|F|_{\beta}=F^{\prime}-F_{\beta}^{\prime}
\end{aligned}
$$

where

$$
D^{\prime}=|D|^{\prime} \quad E^{\prime}=|E| \quad F^{\prime}=|F|
$$

From lemma 2, we have

$$
\left|\operatorname{offdiag}\left(\left(E_{\beta}+G\right) P^{-1}\left(F_{\beta}+H\right)\right)\right| \leq \operatorname{offdiag}\left(\left(E_{\beta}^{\prime}+G^{\prime}\right) P^{-1}\left(F_{\beta}^{\prime}+H^{\prime}\right)\right)
$$

and therefore also

$$
\left|\operatorname{offdiag}\left(\left(E_{\beta}+G\right) P^{-1}\left(F_{\beta}+H\right)\right)-(G+H)\right| \leq \operatorname{offdiag}\left(\left(E_{\beta}^{\prime}+G^{\prime}\right) P^{-1}\left(F_{\beta}^{\prime}+H^{\prime}\right)\right)-\left(G^{\prime}+H^{\prime}\right)
$$

since the two members of inequality (90) are the restrictions of the two members of inequality (89) to the complementary set of $W$ with respect to the whole set of off-diagonal entries.

Thus

$$
|N| \leq D_{\beta}^{\prime}-D^{\prime}+E^{\prime}-E_{\beta}^{\prime}+F_{j}^{\prime}-F_{\beta}^{\prime}+\operatorname{offdiag}\left(\left(E_{\beta}^{\prime}+G^{\prime}\right) P^{-1}\left(F_{\beta}^{\prime}+H^{\prime}\right)\right)-\left(G^{\prime}+H^{\prime}\right)=N^{\prime}
$$

From the relations (80) and (91), we obtain

$$
\left|M^{-1} N\right| \leq\left|M^{-1}\right||N| \leq M^{-1} N^{\prime}
$$

Therefore, by theorem 7 ,

$$
\rho\left(M^{-1} N\right) \leq \rho\left(M^{-1} N^{\prime}\right)
$$


and, by theorem 6 of [1],

$$
\rho\left(M^{-1} N^{\prime}\right)<1
$$

which completes the proof.

Remarks:

$1 \%$ For practical applications, this theorem brings us back to the consideration of sufficient conditions which insure that a given essentially non-positive matrix is an M-matrix. As stated above, this problem has being considered in much detail by Fiedler and Pták and we refer the reader to ref. [3].

$2 \%$ The results given by theorem 7 for general matrices are weaker than the corresponding results given by theorem 6 of [1] for M-matrices since no intercomparison between the convergence rates is given. However combining these results together, it is seen that for general matrices the spectral radius of the associated iteration matrix has an upper bound which is non-increasing with $\beta_{i j}$ for $(i, j) \in V$, nondecreasing with $B_{i i}$ and non-increasing with $W$, when $(P(A))^{-1} \geq 0$, $0 \leq \beta_{i j} \leq 1$ for $(i, j) \in V$ and $\beta_{i i} \geq 1$. 


\subsection{Strict comparisons of asymptotic convergence rates}

Comparisons of the asymptotic convergence rates of two factorization iterative methods were based in [1] on the following theorem of Woznicki [11]

\section{Theorem 9}

Let $A=M_{1}-N_{1}=M_{2}-N_{2}$ be two regular splittings of $A$ where $A^{-1} \geq 0$. If $\mathrm{M}_{1}^{-1} \geq \mathrm{M}_{2}^{-1}$ equality excluded, then

$$
\rho\left(M_{1}^{-1} N_{1}\right) \leq \rho\left(M_{2}^{-1} N_{2}\right)<1
$$

the inequality being strict when $\mathrm{A}^{-1}>0$ and $\mathrm{M}_{1}^{-1}>\mathrm{M}_{2}^{-1}$.

We have the following extension of this result:

Theorem 10

Let $A=M_{1}-N_{1}=M_{2}-N_{2}$ be two regular splittings of $A$ where $A^{-1} \geq 0$. If $\mathrm{M}_{1}^{-1} \geq \mathrm{M}_{2}^{-1}$ equality excluded, then

$$
\rho\left(M_{1}^{-1} N_{1}\right) \leq \rho\left(M_{2}^{-1} N_{2}\right)<1
$$

the inequality being strict when $A^{-1}>0$ and

$$
N_{1}\left(M_{1}^{-1}-M_{2}^{-1}\right) N_{2} \neq 0
$$

or

$$
\mathrm{N}_{2}\left(\mathrm{M}_{1}^{-1}-\mathrm{M}_{2}^{-1}\right) \mathrm{N}_{1} \neq 0
$$

or

$$
\mathrm{N}_{2} \neq \mathrm{N}_{1}=0
$$

$\underline{\text { Proof }}$

As observed by Woznicki [1], the relation

$$
\mathrm{A}^{-1}\left(\mathrm{~N}_{2}-\mathrm{N}_{1}\right) \mathrm{A}^{-1}>0(\text { resp } \geq)
$$

implies

$$
\rho\left(T_{1}\right)<\rho\left(T_{2}\right)<1 \quad\left(\operatorname{resp} \rho\left(T_{1}\right) \leq \rho\left(T_{2}\right)<1\right)
$$

where $T_{i}=M_{i}^{-1} N_{i}$.

If $\mathrm{N}_{2} \neq \mathrm{N}_{1}=0$, we have

$$
A^{-1}\left(N_{2}-N_{1}\right) A^{-1}=A^{-1} N_{2} A^{-1}>0
$$

(1) This theorem was incorrectly reported in [1]; theorem 3 to 8 of [1] must be corrected accordingly. 
when $A^{-1}>0$, thus $\rho\left(T_{1}\right)<\rho\left(T_{2}\right)<1$.

On the other hand, the following identity [11]

$$
\begin{aligned}
A^{-1}\left(N_{2}-N_{1}\right) A^{-1}=\left(M_{1}^{-1}-M_{2}^{-1}\right) & +A^{-1} N_{1}\left(M_{1}^{-1}-M_{2}^{-1}\right)+\left(M_{1}^{-1}-M_{2}^{-1}\right) N_{2} A^{-1} \\
& +A^{-1} N_{1}\left(M_{1}^{-1}-M_{2}^{-1}\right) N_{2} A^{-1}
\end{aligned}
$$

shows that, if $N_{1}\left(M_{1}^{-1}-M_{2}^{-1}\right) N_{2} \neq 0$, then $A^{-1}\left(N_{2}-N_{1}\right) A^{-1}>0$; thus, again, $\rho\left(\mathrm{T}_{1}\right)<\rho\left(\mathrm{T}_{2}\right)<1$.

The last case is proved similarly, using the identity

$$
\begin{aligned}
A^{-1}\left(N_{2}-N_{1}\right) A^{-1}= & \left(M_{1}^{-1}-M_{2}^{-1}\right)+A^{-1} N_{2}\left(M_{1}^{-1}-M_{2}^{-1}\right)+\left(M_{1}^{-1}-M_{2}^{-1}\right) N_{1} A^{-1} \\
& +A^{-1} N_{2}\left(M_{1}^{-1}-M_{2}^{-1}\right) N_{1} A^{-1}
\end{aligned}
$$

In the applications of the latter result, we shall also need the following property of monotone matrices

\section{Theorem 11}

If $\mathrm{A}$ is monotone with positive inverse, then $\mathrm{A}$ is irreducible.

\section{$\underline{\text { Proof }}$}

Indeed, if $A$ is reducible, then using its normal form, it is immediately seen that $A^{-1}$ is also reducible, thus not positive.

As it is well known (see Varga [7]), an irreducible M-matrix has a positive inverse; thus we have

\section{Corol1ary}

Let $A$ be an M-matrix; then $A^{-1}>0$ if and only if $A$ is irreducible.

The following result is a complement of theorem 6 of [1]:

\section{Theorem 12}

Let $A$ be an M-matrix, let $\beta$ and $T$ be the matrix of parameters and the iteration matrix of the Oliphant- Buleev-Woznicki method.

If $0 \leq \beta_{i j}<1$ for $(i, j) \in V, \beta_{i i} \geq 1$ for $i \in[1, n]$, and $A^{-1}>0$, then $\rho(T)$ is a decreasing function of $B_{i j}$ for $(i, j) \in V$, an increasing function 
of $B_{i i}$ and a decreasing function of $W$.

Proof

Assume that

$$
A=M-N=M^{*}-N^{*}
$$

are the splittings corresponding to the Oliphant-Buleev-Woznicki methods applied to $A$ with $B, B^{\prime}$ and $W, W^{\prime}$ as matrices of parameters and sets of off-diagonal entries respectively.

As observed in $[1]$ (with $0 \leq \beta_{i j}, \beta_{i j} \leq 1$ for $(i, j) \in V$ and $\left.\beta_{i i}, \beta_{i i}^{\prime} \geq 1\right)$ if

$$
\begin{aligned}
& B_{i j} \leq B_{i j}^{\prime} \quad \text { for } \quad(i, j) \in V \\
& \beta_{i i} \geq \beta_{i i}^{\prime} \quad \text { for } \quad i \in[1, n] \\
& \mathrm{W} \subset \mathrm{W}^{*}
\end{aligned}
$$

and if one of these relations holds equality excluded, then

$$
M^{-1} \geq M^{-1} \text { equality excluded }
$$

On the other hand, since $A^{-1}>0, A$ is irreducible; thus, if $0 \leq B_{i j} \leq B_{i j}<1$ for $(i, j) \in V$, then $N$ and $N^{\prime}$ are also irreducible; therefore

$$
N^{\prime}\left(M^{-1}-M^{-1}\right) N \neq 0
$$

since the graphs of $N$ and $N^{\prime}$ are strongly connected; thus $\rho\left(T^{\prime}\right)<\rho(T)<1$ by theorem 10 .

\section{$\underline{\text { Remark }}$}

This property does not extend to the complete range $0 \leq \beta_{i j} \leq 1$ for (i, j) $\in V$ without additional assumptions, as shown by the following example

$$
A=\left[\begin{array}{rrr}
1 & 0 & -1 \\
-1 & 1 & 0 \\
0 & -1 & 2
\end{array}\right] \quad \beta=\left[\begin{array}{rrr}
0 & 0 & 0 \\
1 & 0 & 0 \\
0 & 1 & 0
\end{array}\right] \quad \beta^{-}=\left[\begin{array}{lll}
0 & 0 & 1 \\
1 & 0 & 0 \\
0 & 1 & 0
\end{array}\right]
$$

We have $A^{-1}>0, \beta \leq B^{\prime}$ with $\beta \neq \beta^{\prime}$; however

$$
\rho(T)=\rho\left(T^{\prime}\right)=\frac{1}{2}
$$




\section{Block methods}

In this and the next sections,all. matrices are assumed to be partitioned into submatrices indexed $i, j$ with $i, j=1,2 \ldots n$, according to one and the same partitioning, the diagonal submatrices being square.

4.1 The block Buleev method.

With the help of the new definition of the point Buleev method, it becomes a straightforward matter to generalize it as well as composite methods including the Buleev scheme to block factorization iterative processes.

The block Buleev method is defined by

$$
\begin{aligned}
& \mathrm{E}_{1}=\mathrm{E} \quad \mathrm{F}_{1}=\mathrm{F} \\
& \mathrm{P}=\mathrm{D}_{\beta}-\text { blockdiag }\left(\mathrm{EP}^{-1} \mathrm{~F}\right) \\
& \mathrm{D}_{\beta}=\left(\beta_{i i} \cdot \mathrm{D}_{i \mathrm{i}}\right)
\end{aligned}
$$

where $\beta_{i i}$ denotes a block of parameters of the same dimensions as $D_{i i}$ and $\beta_{i i} \cdot D_{i i}$ denotes the element by element product. ${ }^{(1)}$ Other notations are taken from section 2 of ref. [1].

The corresponding splitting is given by

$$
\begin{aligned}
& M=(P-E) P^{-1}(P-F) \\
& N=D_{\beta}-D+\text { offblockdiag }\left(E P^{-1} F\right)
\end{aligned}
$$

\subsection{Composite methods}

The composite block methods of Oliphant-Buleev, Buleev-Woznicki, and 0liphant-Buleev-Woznicki are introduced in the same way. It will be sufficient to quote their definitions, using the notations of ref [1]

The block Oliphant-Buleev method

$$
\begin{aligned}
& E_{1}=E_{\beta} \quad F_{1}=F_{\beta} \\
& P=D_{\beta}-\text { blockdiag }\left(E_{\beta} P^{-1} F_{\beta}\right)
\end{aligned}
$$

(1) better known in the literature as the Hadamard product (cf Styan [15]). 


$$
\begin{array}{ll}
D_{\beta}=\left(\beta_{i j} \cdot A_{i i}\right) & i \in[1, n] \\
E_{\beta}=-\left(\beta_{i s} \cdot A_{i s}\right) & (i, s) \in V_{E} \\
F_{\beta}=-\left(\beta_{s j} \cdot A_{S j}\right) & \left(s_{, j} j\right) \in V_{F} \\
M=\left(P-E_{\beta}\right) P^{-1}\left(P-F_{\beta}\right) & \\
N=\left(D_{\beta}-D\right)+\left(E-E_{\beta}\right)+\left(F-F_{\beta}\right)+\operatorname{offblockdiag}\left(E_{\beta} P^{\left.-1_{F}\right)}\right.
\end{array}
$$

The block Buleev-Woznicki method

$$
\begin{aligned}
& E_{1}=E+G \quad F_{1}=F+H \\
& P=D_{\beta}-\operatorname{blockdiag}\left((E+G) P^{-1}(F+H)\right) \\
& G+H=\operatorname{rest}_{W}\left((E+G) P^{-1}(F+H)\right) \\
& D_{\beta}=\left(\beta_{i i} \cdot A_{i i}\right) \quad i \in[1, n] \\
& M=(P-E-G) P^{-1}(P-F-H) \\
& N=(D-D)+\left(\text { offblockdiag }\left((E+G) P^{-1}(F+H)\right)-(G+H)\right)
\end{aligned}
$$

The block O1iphant-Buleev-Woznicki method

$$
\begin{aligned}
& E_{1}=E_{B}+G \quad F_{1}=F_{B}+H \\
& P=D_{\beta}-\operatorname{blockdiag}\left(\left(E_{\beta}+G\right) P^{-1}\left(F_{\beta}+H\right)\right) \\
& G+H=\operatorname{rest}_{W}\left(\left(E_{\beta}+G\right) P^{-1}\left(F_{\beta}+H\right)\right) \\
& D_{B}=\left(\beta_{i i} \cdot A_{i i}\right) \quad i \in[1, n] \\
& E_{\beta}=-\left(\beta_{i s} \cdot A_{i s}\right) \quad(i, s) \in V_{E} \\
& F_{\beta}=-\left(\beta_{s j} \cdot A_{s j}\right) \quad(s, j) \in V_{F} \\
& M=\left(P-E_{\beta}-G\right) P^{-1}\left(P-F_{\beta}-H\right) \\
& \left.N=\left(D_{\beta}-D\right)+\left(E-E_{\beta}\right)+\left(F-F_{\beta}\right)+\left(\text { offblockdiag }\left(E_{\beta}+G\right) P^{-1}\left(F_{\beta}+H\right)\right)-(G+H)\right)
\end{aligned}
$$


5. Factorizability conditions and convergence criteria for block methods

In order to avoid tedjous repetitions, the notations of section 4 will be used in this and the next section without explicit reference.

\subsection{Essentially non-positive matrices}

We have the following extension of theorem 8 of [1].

Theorem 13

Let $A$ be essentially non-positive, let the entries of $\beta_{i j}$ for $(i, j) \in V$ belong to $[0,1]$, the diagonal entries $b_{k k}^{i 1}$ of $\beta_{i i}$ belong to $[1, \infty]$ and the offdiagonal entries $b_{k l}^{i i}$ of $\beta_{i i}$ belong to $\left[0, b_{k k}^{i}\right]$. Then, the following conditions

(1) $\mathrm{P}$ is an M-matrix;

(2) the block Oliphant-Buleev-Woznicki method is convergent; are simultaneously satisfied if and only if $A$ is an M-matrix.

Under the same assumptions, if $A$ is an $M$-matrix and $T=M^{-1} N$ is the associated iteration matrix, then $\rho(T)$ is

(1) a non-increasing function of $\beta_{i j}$ for $(i, j) \in V$;

(2) a non-decreasing function of the diagonal entries of $B_{i i}$;

(3) a non-increasing function of the off-diagonal entries of $\beta_{i i}$ when its diagonal entries are kept constant;

(4) a non-decreasing function of ${ }^{\theta_{i}}$ when all entries of $\beta_{i i}$ are set equal to $\theta_{i}$

(5) a non-increasing function of $W$.

If, moreover, $A^{-1}>0,\left(\beta_{i j} \cdot D_{i i}\right)^{-1}>0$ for $i \in[1, n]$, and $\{1\}$ is deleted from the ranges of the off-diagonal parameters, then $\rho(\mathrm{T})$ is:

(1) a decreasing function of $\beta_{i j}$ for $(i, j) \in V$;

(2) an increasing function of the diagonal entries of $\beta_{i i}$;

(3) a decreasing function of the off-diagonal entries of $\beta_{i i}$ when its diagonal entries are kept constant;

(4) an increasing function of $A_{i}$ when all entries of $\beta_{1 i}$ are set equal to $\theta_{i} ;$ 
(5) a decreasing function of $W$.

\section{Proof}

We first show that under the general assumptions of the theorem, if $P$ is an M-matrix, then the splitting

$$
A=M-N
$$

which describes the block 01iphant-Buleev-Woznicki method is a weak regular splitting.

We have successively

$$
\begin{aligned}
& M=\left(I-\left(E_{B}+G\right) P^{-1}\right)\left(I-\left(F_{\beta}+H\right) P^{-1}\right) P \\
& M^{-1}=P^{-1}\left(I-\left(F_{\beta}+H\right) P^{-1}\right)^{-1}\left(I-\left(E_{B}+G\right) P^{-1}\right)^{-1} \\
& M^{-1}=P^{-1}\left(\sum_{k=0}^{n-1}\left(\left(F_{\beta}+H\right) P^{-1}\right) k\right)\left(\sum_{l=0}^{n-1}\left(\left(E_{\beta}+G\right) P^{-1}\right)^{l}\right)
\end{aligned}
$$

If $P$ is an $M$-matrix, we have

$$
\mathrm{P}^{-1} \geq 0
$$

and this relation, together with

$$
G+H=\operatorname{rest}_{W}\left(\left(E_{B}+G\right) P^{-1}\left(F_{B}+H\right)\right)
$$

and

$$
E_{B} \geq 0 \quad F_{B} \geq 0
$$

entails

$$
G \geq 0 . \quad H \geq 0
$$

(by induction on the successive columns of $G$ and rows of $H$ ). Thus

$$
\mathrm{M}^{-1} \geq 0
$$

On the other hand, the relation

$$
P=D_{\beta}-\text { blockdiag }\left(\left(E_{B}+G\right) P^{-1}\left(F_{B}+H\right)\right)
$$

together with (141), (143) and (144) entails

$$
P \leq D_{B}
$$

Thus $D_{\beta}$ is also an M-matrix (see theorem 1 of [1]) and

$$
\mathrm{D}_{\mathrm{B}} \mathrm{P}^{-1} \geq \mathrm{I}
$$


Consider now

$$
N=\left(D_{B}-D\right)+\left(E-E_{B}\right)+\left(F-F_{B}\right)+\left(\text { off block diag }\left(\left(E_{B}+G\right) P^{-1}\left(F_{B}+H\right)\right)-(G+H)\right)
$$

and let $B$ be the purely diagonal matrix $\left(b_{k k}^{i j}\right)$ :

$$
B=\operatorname{diag}(\beta)
$$

We have

$$
\begin{aligned}
& \mathrm{D}_{\beta} \geq \mathrm{BD} \\
& \mathrm{I} \geq \mathrm{B}^{-1} \geq \mathrm{DD}_{\beta}^{-1}
\end{aligned}
$$

Therefore, by (148),

$$
\left(D_{\beta}-D\right) P^{-1}=\left(I-D D_{\beta}^{-1}\right) D_{\beta} P^{-1} \geq 0
$$

and, thus

$$
\left(D_{B}-D\right) M^{-1} \geq 0
$$

Since the entries of $\beta_{i j}$ for $(i, j) \in V$ belong to $[0,1]$, we have

$$
\left(E-E_{B}\right) \geq 0 \quad\left(F-F_{B}\right) \geq 0
$$

thus also

$$
\left(E-E_{B}\right) M^{-1} \geq 0 \quad\left(F-F_{\beta}\right) M^{-1} \geq 0
$$

and it follows from (141), (142), (143) and (144) that the last term of the right hand side of (149) is also nonnegative; thus

$$
\mathrm{NM}^{-1} \geq 0
$$

and the splitting (137) is a weak regular splitting.

Therefore, if $\mathrm{P}$ is an M-matrix and the block 0liphant-Buleev-Woznicki method is convergent in the assumed range for the iteration parameters, then A is an M-matrix by theorem 1 .

Conversely, if $\mathrm{A}$ is an M-matrix, then

$$
A_{B}=D_{B}-E_{B}-F_{B} \geq B D-E-F \geq B A
$$

is also an M-matrix (see theorem 1 of [1]), therefore $\mathrm{P}$ is an M-matrix by lemma 6 of [1] and the splitting (137) is a weak regular splitting; thus $\rho(\mathrm{T})<1$ by theorem 1 .

For the second part of the theorem, we observe that, if $\mathrm{A}$ is an M-matrix, then 


$$
\mathrm{AD}^{-1}=\mathrm{MD}^{-1}-\mathrm{ND}^{-1}
$$

is a regular splitting of $\mathrm{AD}^{-1}$.

We have indeed

$$
D M^{-1}=\left(\sum_{k=0}^{n-1}\left(D P^{-1}\left(F D^{-1}+H D^{-1}\right)\right)^{k}\right)\left(\sum_{\ell=0}^{n-1}\left(D P^{-1}\left(E_{\beta} D^{-1}+G D^{-1}\right)\right)^{l}\right) D P^{-1}
$$

and

$$
\mathrm{PD}^{-1}=\mathrm{D}_{\beta} \mathrm{D}^{-1}-\operatorname{blockdiag}\left(\left(\mathrm{E}_{\beta} \mathrm{D}^{-1}+\mathrm{GD}^{-1}\right) \mathrm{DP}^{-1}\left(\mathrm{~F}_{\beta} \mathrm{D}^{-1}+\mathrm{HD}^{-1}\right)\right)
$$

By (158), we have

$$
\mathrm{A}_{\beta} \mathrm{D}^{-1} \geq \mathrm{B}\left(\mathrm{AD}^{-1}\right)
$$

Thus $A_{B} D^{-1}$.is an M-matrix by theorem 1 of [1] and so is $\mathrm{PD}^{-1}$ by lemma 6 of [1]. Therefore, using (143) and (144),

$$
\mathrm{DM}^{-1} \geq 0
$$

On the other hand, we have, by (151)

$$
\begin{aligned}
& \mathrm{D}_{\beta} \mathrm{D}^{-1} \geq \mathrm{B} \geq \mathrm{I} \\
& \mathrm{D}_{\beta} \mathrm{D}^{-1}-\mathrm{I} \geq 0
\end{aligned}
$$

and, therefore, using (149)

$$
\mathrm{ND}^{-1} \geq 0
$$

(1) Let $\beta$ and $\beta^{\prime}$ satisfy the general assumptions of the theorem with

$$
\begin{aligned}
\beta_{i i}=\beta_{i i}^{\prime} \text { and } & \\
& \beta_{i j} \leq \beta_{i j}^{\prime} \text { for all }(i, j) \in V
\end{aligned}
$$

Then

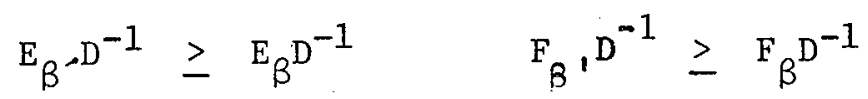

and, by lemma 6 of [1]

$$
\mathrm{DP}^{\prime-1} \geq \mathrm{DP}^{-1}, \mathrm{G}^{\prime} \mathrm{D}^{-1} \geq \mathrm{GD}^{-1}, \quad \mathrm{H}^{\prime} \mathrm{D}^{-1} \geq \mathrm{HD}^{-1}
$$

thus, by (160),

$$
\mathrm{DM}^{\prime-1} \geq \mathrm{DM}^{-1}
$$

Therefore, by theorem 9 ,

$$
\rho\left(D M^{1}-1 N^{\prime} D^{-1}\right)=\rho\left(M^{1}-1 N^{\prime}\right) \leq \rho\left(D M^{-1} N D^{-1}\right)=\rho\left(M^{-1} N\right)
$$


We have similarly, by (140),

$$
\mathrm{H}^{-1} \geq \mathrm{M}^{-1}
$$

and, moreover if equality is excluded in (167) and if $\left(\beta_{i i} D_{i i}\right)^{-1}>0$ for $i \in[1, n]$, then equality is excluded in (172) since $\mathrm{P}_{i i}^{-1}>0$ for $i \in[1, n]$ by. $(147)$.

On the other hand $\mathrm{A}^{-1}>0$ implies that $\mathrm{A}$ is irreducible by theorem 11 ; if the off-diagonal parameters (the entries of $\beta_{i j}$ for $(i, j) \in V$ and the elements $b_{k l}^{i i}$ for $k \neq l$ ) are $\neq 1$, then $N$ and $N^{\prime}$ are also irreducible, therefore, $N^{\prime}\left(M^{\prime-1}-M^{-1}\right) N \neq 0$

thus also

$$
N^{\prime} D^{-1}\left(D M^{1-1}-D M^{-1}\right) N^{-1} \neq 0
$$

and

$$
\rho\left(D M^{\prime-1} N^{\prime} D^{-1}\right)=\rho\left(M^{\prime-1} N^{\prime}\right)<\rho\left(D^{-1} N D\right)=\rho\left(M^{-1} N\right)
$$

by theorem 12 .

(2) and (3) Let $\beta$ and $\beta^{\prime}$ satisfy the general assumptions of the theorem with $\beta_{i j}=B_{i j}^{\prime}$ for all $(i, j) \in V$ and

$$
\beta_{i i} \cdot D_{i i} \geq \beta_{i i}^{\prime} \cdot D_{i i} \text { for } i \in[1, n]
$$

i.e.

$$
D_{B} \geq D_{B}
$$

Then

$$
\begin{aligned}
& D_{B D^{-1}} \geq D_{B} \cdot D^{-1} \\
& A_{B} D^{-1} \geq A_{B} \cdot D^{-1}
\end{aligned}
$$

and, by lemma 6 of [1],

$$
\mathrm{DP}^{1-1} \geq \mathrm{DP}^{-1}, \quad \mathrm{G}^{\prime} \mathrm{D}^{-1} \geq \mathrm{GD}^{-1}, \quad \mathrm{H}^{\prime} \mathrm{D}^{-1} \geq \mathrm{HD}^{-1}
$$

As in the first case, we have then

$$
\rho\left(M^{\prime-1} N^{\prime}\right) \leq \rho\left(M^{-1} N\right)
$$

We have simflarly 


$$
M^{\prime-1} \geq M^{-1}
$$

and, moreover, if equality is excluded in (176), then

$$
\mathrm{P}^{\prime} \leq \mathrm{P} \text { with } \mathrm{P}^{\prime} \neq \mathrm{P}
$$

and, thus, equality is excluded in (182); the proof is concluded as in the first case.

(4) In this case we have

$$
P D^{-1}=B-\operatorname{blockdiag}\left(\left(E_{\beta} D^{-1}+G^{-1}\right) D P^{-1}\left(F_{\beta} D^{-1}+H^{-1}\right)\right)
$$

Again, by lemma 6 of $[1]$, if

$$
I \leq B^{\prime} \leq B
$$

we have

$$
\mathrm{DP}^{\prime-1} \geq \mathrm{DP}^{-1}, \quad \mathrm{G}^{\prime} \mathrm{D}^{-1} \geq \mathrm{GD}^{-1}, \quad \mathrm{H}^{\prime} \mathrm{D}^{-1} \geq \mathrm{HD}^{-1}
$$

thus also (170) and (171).

On the other hand

$$
P=B D-\operatorname{blockdiag}\left(\left(E_{\beta}+G\right) P^{-1}\left(F_{B}+H\right)\right)
$$

and

$$
G+H=\operatorname{rest}_{W}\left(\left(E_{\beta}+G\right) P^{-1}\left(F_{\beta}+H\right)\right)
$$

Then it is seen, by induction on the block entries of $P, G$ and $H$ that

$$
B^{\prime} \leq B^{\prime} \text { with } B^{\prime} \neq B
$$

implies

$$
P^{\prime} \leq P \text { with } P^{\prime} \neq P, G^{\prime} \geq G, H^{\prime} \geq H
$$

and thus

$$
M^{1^{-1}} \geq M^{-1} \quad \text { with } M^{1^{-1}} \neq M^{-1}
$$

The proof is concluded as in the first case.

$$
\begin{aligned}
& \text { (5) Let } \\
& W \subset W^{\prime}
\end{aligned}
$$

Then, by lemma 6 of [1],

$$
\mathrm{DP}^{-1} \geq \mathrm{DP}^{-1}, \quad \mathrm{G}^{\prime} \mathrm{D}^{-1} \geq \mathrm{GD}^{-1}, \quad \mathrm{H}^{\prime} \mathrm{D}^{-1} \geq \mathrm{HD}^{-1}
$$


thus also (170) and (171).

We have similarly, by (140)

$$
M^{1-1} \geq M^{-1}
$$

and, moreover, if equality is excluded in (192) and if $\left(\beta_{i i} \cdot D_{i i}\right)^{-1}>0$

for $i \in[1, n]$, then equality is excluded in (194) since $\mathrm{P}_{i i}^{-1}>0$ for

$i \in[1, \mathrm{n}]$ by $(147)$.

The proof is concluded as in the first case.

Remark

We may equivalently assume, in theorem 9 that the off-diagonal entries $b_{k \ell}^{i i}$ of $\beta_{i i}$ belong to $\left[0, b_{l \ell}^{i i}\right]$; the proof is similar, showing that under the general assumptions of the theorem, if $P$ is an M-matrix then $M^{-1} \geq 0$ and $M^{-1} \mathrm{~N} \geq 0$. The last part of the the theorem is proved in this case by showing that if $A$ is an M-matrix,

$$
\mathrm{D}^{-1} \mathrm{~A}=\mathrm{D}^{-1} \mathrm{M}-\mathrm{D}^{-1} \mathrm{~N}
$$

is a regular splitting of $\mathrm{D}^{-1} \mathrm{~A}$, and by applying a similar reasoning.

\subsection{Further results on the characterization of M-matrices}

\subsection{The block Choleski factorization of M-matrices}

We have the following extension of lemma 1:

Lemma 3

Let $A=K-L-U$ where $K, L$ and $U$ are respectively block-diagonal, strictly lower and upper block-triangular matrices, be essentially nonpositive and let the block-diagonal, strictly lower and upper blocktriangular matrices $P, G$ and $H$ respectively be defined by

$$
\begin{aligned}
& \mathrm{P}=\mathrm{K}-\operatorname{block-diag}\left((\mathrm{L}+\mathrm{G}) \mathrm{P}^{-1}(\mathrm{U}+\mathrm{H})\right) \\
& \mathrm{G}+\mathrm{H}=\operatorname{offblock-diag}\left((\mathrm{L}+\mathrm{G}) \mathrm{P}^{-1}(\mathrm{U}+\mathrm{H})\right)
\end{aligned}
$$

Then $\mathrm{P}$ is an M-matrix if and only if $\mathrm{A}$ is an M-matrix. 


\section{Proof}

For the sufficient condition, we refer to $[1]$; the necessary condition is a straightforward extension of the proof of lemma 1 and we refer to section 32.1 .

Similarly, we have the following extension of theorem 4:

\section{Theorem. 14}

Let $A=K-L-U$ where $K, L$ and $U$ are respectively block-diagonal, strictly lower and upper block-triangular matrices be essentially nonpositive ${ }^{(1)}$ and let $\mathrm{D}_{i}$ be its leading principal minors relative to the same partitioning; let the block-diagonal, strictly lower and upper block triangular matrices $P, G$ and $H$ respectively be defined by

$$
\begin{aligned}
& P=K-\operatorname{block-diag}\left((L+G) P^{-1}(U+H)\right) \\
& G+H=\operatorname{rest}_{W}\left((L+G) P^{-1}(U+H)\right)
\end{aligned}
$$

where $W$ is an arbitrary set of off-diagonal indices.

If $A$ is an M-matrix, $P$ is an M-matrix and

$$
D_{i} \leq \prod_{s=1}^{i} \operatorname{det}\left(P_{s s}\right)
$$

with equality if $W$ contains the block-Choleski set of $A$, for the same partitioning.

\section{Proof}

From lemma 6 of [1], $P$ is an M-matrix and, as in the proof of theorem 4, we have

$$
D_{i}=\prod_{s=1}^{i} \operatorname{det}\left(P_{s s}\right)
$$

when $W$ contains the block-Choleski set of A.

From lemma 6 of [1] we know that $P$ is a non-increasing function of the set $W$ and the conclusion follows from the corollary of theorem 4.

(1) i. e., $\mathrm{L} \geq 0, \mathrm{U} \geq 0$ and $\mathrm{K}$ essentially non positive. 


\subsection{A further characterization of M-matrices}

We have the following extension of theorem 6:

Theorem 15

Let $A$ be an essentially non-positive matrix. Then $A$ is an $M$-matrix if and only if $A$ may be written in the form $R S$ where $R$ and $S$ are respectively lower and upper block-triangular M-matrices according to one and the same (but otherwise arbitrary) partitioning.

\section{$\underline{\text { Proof }}$}

If $\mathrm{A}$ is an M-matrix, the conclusion follows from lemma 3 by putting

$$
\begin{aligned}
& R=P-L-G \\
& S=P^{-1}(P-U-H)
\end{aligned}
$$

If, conversely, we have

$$
A=R S
$$

where $\mathrm{R}$ and $\mathrm{S}$ are $\mathrm{M}$-matrices, then

$$
A^{-1}=S^{-1} R^{-1}
$$

is monotone since $S^{-1} \geq 0$ and $R^{-1} \geq 0$.

\subsection{Criteria for general matrices}

We have the following extension of lemma 2 to partitioned matrices:

\section{Lemma 4}

Let $A=K-L-U$ where $K, L$ and $U$ are respectively block-diagonal, strictly lower and upper block-triangular be an arbitrary partitioned matrix; let the block-diagonal, strictly lower and upper block-triangular matrices $P, G$ and $H$ respectively be defined by

$$
\begin{aligned}
& P=K-\operatorname{block-diag}\left((L+G) P^{-1}(U+H)\right) \\
& G+H=\operatorname{rest}_{W}\left((I+G) P^{-1}(U+H)\right)
\end{aligned}
$$

where $W$ is an arbitrary set of off-block-diagonal entries; let 
$P(A)=K^{\prime}-L^{\prime}-U^{\prime}$ be the decomposition of $P(A)$ in its block-diagonal, strictly lower and upper block-triangular parts according to the same partitioning and let $P^{\prime}, G^{\prime}$ and $H^{\prime}$ be defined by (206) and (207) applied to' $\mathrm{K}^{\prime}, \mathrm{L}^{\prime}$ and $\mathrm{U}^{\prime}$.

If $P(A)$ is an M-matrix, then $P$ is non-singular; we have moreover

$$
\left|P^{-1}\right| \leq P^{-1} \quad|G| \leq G^{\prime} \quad|H| \leq H^{\prime}
$$

Proof

Let $P_{i i}, K_{i i}, L_{i s}, G_{i s}, U_{s j}$ and $H_{s j}$ denote the block-entries of the partitioned matrices $P, K, L, G, U$ and $H$ respectively; the relation defining $P, G$ and $H$ may be written

$$
\begin{aligned}
& P_{i i}=K_{i i}-\sum_{s=1}^{i-1}\left(L_{i s}+G_{i s}\right) P_{s s}^{-1}\left(U_{s i}+H_{s i}\right) \\
& \dot{G}_{k i}= \begin{cases}\sum_{s=1}^{i-1}\left(L_{k s}+G_{k s}\right) P_{s s}^{-1}\left(U_{s i}+H_{s i}\right) & \text { if }(k, i) \in W \\
0 & \text { if }(k, i) \notin W\end{cases} \\
& H_{i k}= \begin{cases}i-1 \\
\sum_{s=1}\left(L_{i s}+G_{i s}\right) P_{s s}^{-1}\left(U_{s k}+H_{s k}\right) & \text { if }(i, k) \in W \\
0 & \text { if }(i, k) \notin W\end{cases}
\end{aligned}
$$

and similar relations hold between $P_{i i}^{\prime}, K_{i i}^{\prime}, L_{i s}^{\prime}, G_{i s}^{\prime}, U_{s j}^{\prime}$ and $H_{s j}^{\prime}$, the block-entries of $\mathrm{P}^{\prime}, \mathrm{K}^{\prime}, \mathrm{L}^{\prime}, \mathrm{G}^{\prime}, \mathrm{U}^{\prime}$ and $\mathrm{H}^{\prime}$.

We show by induction that

$$
\begin{aligned}
& P\left(P_{s s}\right) \geq P_{s s}^{\prime} \\
& \left|G_{k s}\right| \leq G_{k s}^{\prime} \text { and }\left|H_{s k}\right| \leq H_{k s}^{\prime} \quad \text { for } k>s
\end{aligned}
$$


For $s=1$, the proposition is evident since

$$
\begin{array}{ll}
P\left(P_{11}\right)=P\left(K_{11}\right)=P_{11}^{\prime} & \\
G_{k 1}=G_{k 1}^{\prime}=0 & k>1 \\
H_{1 k}=H_{1 k}^{\prime}=0 & k>1
\end{array}
$$

Assume that these relations hold for $s, i-1$; then applying the corollary of lemma 2 to the induction hypothesis (212), we conclude that $P_{\text {sS }}$ is not singular and

$$
\left|\mathrm{P}_{s s}^{-1}\right| \leq\left(\mathrm{P}\left(\mathrm{P}_{\mathrm{SS}}\right)\right)^{-1}
$$

and, by theorem 1 of $[1]$,

$$
\left(P\left(P_{S S}\right)\right)^{-1} \leq P_{S S}^{-1}
$$

Thus, the relation (210) is meaningful and we have successively, for $k>i, \quad(k, i) \in W$

$$
\left|G_{k i}\right| \leq \sum_{s=1}^{i-1}\left(\left|I_{k s}\right|+\left|G_{k s}\right|\right)\left|P_{s s}^{-1}\right|\left(\left|U_{s i}\right|+\left|H_{s i}\right|\right)
$$

or

$$
\left|G_{k i}\right| s \sum_{s=1}^{i=1}\left(L_{k s}^{\prime}+\left|G_{k s}\right|\right)\left|P_{s s}^{-1}\right|\left(U_{s i}^{\prime}+\left|H_{s i}\right|\right)
$$

and, applying (217), (218) and (213),

$$
\left|G_{k i}^{\prime}\right| \leq \sum_{s=1}^{i-1}\left(L_{k s}^{\prime}+G_{k s}^{\prime}\right) P_{s s}^{-1}\left(U_{s i}^{\prime}+H_{s i}^{\prime}\right)=G_{k i}^{\prime}
$$

On the other hand, if $k>i$ and $(k, i) k W$, we have

$$
G_{k i}=G_{k i}^{\prime}=0
$$

The same method of proof applies to $H_{i k}$ for $k>i$, and to the offdiagonal elements of $P_{i i}$, thus showing that

$$
\text { offdiag }\left|P_{i i}\right| \leq \operatorname{offdiag}\left|P_{i i}^{\prime}\right|
$$


For the diagonal elements of $\mathrm{P}_{i i}$, we have

$$
\operatorname{diag}\left|P_{i i}\right| \geq \operatorname{diag}\left|K_{i i}\right|-\sum_{S=1}^{i-1} \operatorname{diag}\left|\left(L_{i s}+G_{i s}\right) P_{s s}^{-1}\left(U_{s i}+H_{s i}\right)\right|
$$

and the same method of proof applies to the second term of the right hand side of this inequality; thus

$$
\operatorname{diag}\left|P_{i i}\right| \geq \operatorname{diag}\left(P_{i i}^{\prime}\right)
$$

and, from (223) and (224) we conclude that

$$
P\left(P_{i i}\right) \geq P_{i i}^{\prime}
$$

Therefore we have

$$
P(P) \geq P^{\prime}
$$

and, applying the corollary of lemma 2 and theorem 1 of $[1]$, we have

$$
\left|\mathrm{P}^{-1}\right| \leq(\mathrm{P}(\mathrm{P}))^{-1} \leq \mathrm{P}^{-1}
$$

Theorem 16

If $P(A)$ is an $M$-inatrix, if the entries of $\beta_{i j}$ for $(i, j) \in V$ belong to $[0,1]$, the diagonal entries $b_{k k}^{i i}$ of $\beta_{i i}$ belong to $[1, \infty]$ and the off-diagonal entries $b_{k l}^{i i}$ of: $\beta_{i i}$ belong to $[0,1]$, then the block-matrix $P$ of the block 01iphant-Buleev-Woznicki method is non-singular and the method is convergent. Moreover its convergence rate is not less than the convergence rate of the same method applied to $P(A)$.

\section{Proof}

Let $A=M-N$ and $P(A)=M^{\prime}-N^{\prime}$ be the splitting of $A$ and $P(A)$ relative to the block Oliphant-Buleev-Woznicki scheme.

As in the proof of theorem 8 , but with $D^{\prime}=P(D)$ and $D_{\beta}^{\prime}=P\left(D_{\beta}\right)=(P(D))_{B}$ it is seen that

$$
\begin{aligned}
& \left|M^{-1}\right| \leq M^{-1} \\
& |N| \leq N^{\prime}
\end{aligned}
$$


Therefore

$$
\left|M^{-1} N\right| \leq N^{-1} N^{\prime}
$$

and, by theorem 7

$$
\rho\left(M^{-1} N\right) \leq \rho\left(M^{-1} N^{\prime}\right)
$$

The conclusion follows from theorem 13 .

Remark

A similar property holds if we assume that the entries of each block $\beta_{i j}$ are all equal to one and the same number $\theta_{i j}$ with $0 \leq \theta_{i j} \leq 1$ for $(i, j) \in V$ and $\theta_{i i} \geq 1$ for $i \in[1, n]$. (cf theorem 21 below). 


\section{Block-M-matrices}

\subsection{An extension of a theorem of Ky Fan}

We investigate here the possible extension of our results for blockmethods to a larger class of matrices, the block-M-matrices, whose definition follows.

We refer to $[17$ for the definition and necessary properties of monotone matrices, M-matrices and regular splittings.

By a $n \times n$ partitioned matrix, we mean any matrix $A=\left(A_{i j}\right)$ partitioned into submatrices $A_{i j}$ with $i, j=1,2, \ldots n$, the diagonal submatrices being square.

\section{$\underline{\text { Definition }}$}

A real $n \times n$ partioned matrix $A=\left(A_{i j}\right)$ with $A_{i j} \leq 0$ for $i \neq j$ is a block-M-matrix if $A$ and $A_{i i}$ are monotone for $i=1,2$. . $n$.

The following theorem gives'a characterization of block-M-matrices; it is an extension of a theorem of Ky Fan $[12\rceil$ and Vandergraft $[10 ?$.

\section{Theorem 17}

Let $A=\left(A_{i j}\right)$ be a real $n \times n$ partitioned matrix such that $A_{i j} \leq 0$ for $i \neq j$. Then $A$ is a block-M-matrix if and only if:

$1 \%$ there exists a non-singular, non-negative, block-diagonal matrix $B$ (according to the same $n \times n$ partitioning) such that $B A \leq I$;

$2 \%$ there exists $x \geq 0$ such that $A x>0$.

$\underline{\text { Remark }}$

Under the hypotheses of the theorem, it is easily seen that the $2^{\circ}$ is equivalent to: there exists $x>0$ such that $A x>0$.

\section{$\underline{\text { Proof }}$}

If $A$ is a block-M-matrix, let $B=K^{-1}$ where

$$
K=b \operatorname{lock}-\mathrm{diag}(\mathrm{A})
$$

On the other hand, let $x=A^{-1} y$ for any $y>0$; since $A^{-1} \geq 0$, we have then $\mathrm{x} \geq 0$ and $\mathrm{Ax}>0$. 
Conversely, if there exists a non-singular $B \geq 0$ such that $B A \leq I$ and a vector $\mathrm{x} \geq 0$ such that $A x>0$, then by applying the interior mapping principle (see $\Gamma 137$, chap. II, section 2.1 ), we have also BAx $>0$; thus

$$
0 \leq(I-B A) x<x
$$

Since $(I-B A) \geq 0$, there exists a non-negative eigenvector $x_{0}$ of (I - BA) associated with $\rho(I-B A)$, by theorem 2.7 of Varga $\Gamma 71$.

We now prove that $\rho(I-B A)<1$; therefore we may assume that $p(I-B A)>0$. Then, following Vandergraft's method of proof $[10]$, let

$$
t_{0}=\sup \left(t>0 ; \quad \operatorname{tx}_{0} \leq x\right)
$$

Since $x$ is strictly positive by $(170)$, such a number $t_{0}$ exists and is finite. Then

$$
\rho(I-B A) t_{0} x_{0}=t_{0}(I-B A) x_{0} \leq(I-B A) x<x
$$

But $t_{0}$ is the supremum of the $t>0$ such that $t_{0} \leq x$; thus

$$
\rho(I-B A) t_{0}<t_{0}
$$

and

$$
\rho(I-B A)<1
$$

Therefore, the Neumann series in powers of ( $I$ - BA) is convergent and defines $(\mathrm{BA})^{-1}$

$$
(\mathrm{BA})^{-1}=\sum_{k=0}^{\infty}(I-B A)^{k}
$$

Since each term of the series is non-negative, we have (BA) $)^{-1} \geq 0$ and, multiplying by $B \geq 0$,

$$
A^{-1}=(B A)^{-1} B \geq 0
$$

Thus A is monotone.

Since $A_{i j} \leq 0$ for $i \neq j$ and $B$ is block-diagonal and non-negative, 
our initial assumptions on $A$ are also true for $K=$ block-diag(A); therefore, the same conclusion holds: $K$ is monotone.

Thus $A$ is a block-M-matrix.

\section{Corollary}

Let $A=\left(A_{i j}\right)$ and $A^{\prime}=\left(A_{i j}^{\prime}\right)$ be real $n \times n$ partitioned matrices such that $A_{i j}^{\prime} \leq 0$ for $i \neq j, A \leq A^{\prime}$ and $A$ is monotone.

Then $A^{\prime}$ is a block-M-matrix if and only if there exists a non-singular, non-negative, block-diagonal matrix $\mathrm{B}$ (according to the same partitioning) such that $\mathrm{BA}^{\prime} \leqslant \mathrm{I}$.

$\underline{\text { Proof }}$

The necessary condition is part of theorem 17; on the other hand, if $\mathrm{A}$ is monotone, then, by applying theorem 17 to the matrix A considered as one block, there exists a vector $\mathrm{x} \geq 0$, such that $A \mathrm{x}>0$.

Since $A \leq A^{\prime}$ we have also

$$
A^{\prime} x \geq A x>0
$$

and the conclusion follows from theorem 17.

$\underline{\text { Remark }}$

As a consequence, if $A=\left(A_{i j}\right)$ is a monotone, $n \times n$ partitioned matrix with $A_{i j} \leq 0$ for $i \neq j$, then $A$ itself is a block-M-matrix if and only if there exists a non-singular, non-negative, block-diagonal matrix $B$ such that $B A \leq I$.

\subsection{Factorizability conditions and convergence criteria}

We have' the following extension of lemma 6 of 517 to the class of block-M-matrices.

Lemma 5

Let $A=K-L-U$ where $K^{-1} \geq 0, L \geq 0$ and $U \geq 0$ are respectively block-diagonal, strictly lower and upper block-triangular matrices and let the block-diagonal, strictly lower and upper block-triangular matrices $P, C$ and $H$ be defined by 


$$
\begin{aligned}
& P=K-\text { blockdiag }\left((L+G) P^{-1}(U+H)\right) \\
& G+H=\operatorname{rest}_{W}\left((L+G) P^{-1}(U+H)\right)
\end{aligned}
$$

where $W$ is a given set of offblockdiagonalblock-entries (deleting zero entries).

If $A$ is a block-M-matrix, $P$ is monotone, and, thus, $G$ sind il are non-negative; moreover $\mathrm{P}$ is a non-decreasing function of $\mathrm{A}$ (as far as A remains a block-M-matrix) and a non-increasing function of $W ; G$ and $H$ are non-increasing function of $A$ (as far as $A$ remains a block-M-matrix) and $G+H$ is an increasing function of $W$.

\section{Proof}

1. The proof is very similar to the proof of lemma 6 of $\lceil 17$ and will. not be reproduced. The only difference consists of showing that the first condition of theorem 17 is satisfied for $P$ whenever it is satisfied for $A$, and this is obvious since $P \leq K$.

As a consequence, we have the following convergence theorem for the block Oliphant-Woznicki method.

\section{Theorem 18}

Let $A=D-E-F$ be a real $n \times n$ partitioned matrix where $D, E$ and $F$ are respectively block-diagonal, strictly lower and upper block-triangular with $E \geq 0$ and $F \geq 0$. Let the entries of the blocks of parameters $\beta_{i j}$, $(i, j) \in V$ of the block 0liphant-Woznicki method belong to $[0,1]$.

Then, the following conditions

(1) D and $P$ are monotone;

(2) the block Oliphant-Woznicki method converges;

are simultaneously satisfied if and only if $A$ is a block-M-matrix. Under the same assumptions, if $A$ is a block-M-matrix and $T$ is the iteration matrix associated with the block Oliphant-Woznicki method, then $\rho(T)$ is a 
non-increasing function of $B_{i . j},(i, j) \in V$ in this range and a non-increasing function of $W$. If moreover $A^{-1}>0, D_{i i}^{-1}>0$ for $i \in[1, n]$ and the entries of $\beta_{i j},(i, j) \in V$ belong to $[0,1[$, then $p(T)$ is a decreasing function of $B_{i j}$ and of $W$.

\section{$\underline{\operatorname{Proof}}$}

The proof is similar to the proof of theorem 13; therefore we shal1 simply indicate the main steps

It is first shown that if the condition ( 1 ) is satisfied, then the splitting

$$
A=M-N
$$

associated with the block Oliphant-Woznicki method is a regular splitting.

The first part of the theorem is then a consequence of theorem 1 , and the last parts are proven by applying theorems 9 and 10.

The extension of this theorem to block methods including the block Buleev scheme will be helped by introducing the following notations. Let $\mathrm{p}_{i i}^{+}, \mathrm{D}_{i i}^{-}$, $\beta_{i i}^{+}$and $\beta_{i i}^{-}$be defined by

$$
\begin{aligned}
& \mathrm{D}_{i i}=\mathrm{D}_{i i}^{+}-\mathrm{D}_{i i}^{-}, \mathrm{D}_{i i}^{+} \geq 0, \mathrm{D}_{i i}^{-} \geq 0 \\
& \beta_{i i}=\beta_{i i}^{+}+\beta_{i i}^{-} \\
& \beta_{i i} \cdot \mathrm{D}_{i i}=\beta_{i i}^{+} \cdot \mathrm{D}_{i i}^{+}-\beta_{i i}^{-} \cdot \mathrm{D}_{i i}^{-}
\end{aligned}
$$

In other words, the non-zero entries of ${D_{i i}^{+}}_{\left(D_{i i}^{-}\right)}^{-}$are the positive (negative). entries of $D_{i i}$; the non-zero entries of $\beta_{i i}^{+}\left(\beta_{i i}^{-}\right)$are the entries of $\beta_{i i}$ which multiply the positive (negative) entries of $\mathrm{D}_{i i}$. We have then:

\section{Theorem 19}

Let $A=D-E-F$ be a real $n \times n$ partitioned matrix where $D$ is blockdiagona1, $E \geq 0$ and $F \geq 0$ are respectively strictly lower and upper blocktriangular; let $B$ and $W$ be the matrix of parameters and the set of off-diagonal 
block-entries associated with the block 01iphant-Buleev-Woznicki method.

Let the entries of the blocks $\beta_{i j},(i, j) \in V$ belong to $[0,1]$; let the entries of $\beta_{i i}^{+}$belong to $[1, \infty]$ and for any line $k$ of the block $\beta_{i i}$, let $b_{k}^{i}$ be a minimal non-zero entry of $\beta_{i i}^{+}$on that line; let the entries of the $k^{\text {th }}$ line of $\beta_{i i}^{-}$belong to $\left[0, b_{k}^{i}\right]$ and assume that $\left(\beta_{i i} \cdot D_{i i}\right)^{-1} \geq 0$.

Then the following conditions

(1) $\mathrm{P}$ is monotone

(2) the block 0liphant-Buleev-Woznicki method is convergent; are simultaneously satisfied if and only if $A$ is a block-M-matrix. Under the same assumptions, if $A$ is a block M-matrix and $T=M^{-1} N$ is the associated iteration matrix, then $\rho(T)$ is

(1) a non-increasing function of $\beta_{i j}$ for $(i, j) \in V$;

(2) a non-decreasing function of $\beta_{i i}^{+}$;

(3) a non-increasing function of $\beta_{i i}^{-}$when $b_{k}^{i}$ is kept constant;

(4) a non-decreasing function of $\theta_{i}$ when all the entries of $\beta_{i i}$ are set equal to $\theta_{i}$;

(5) a non-increasing function of $W$.

If moreover $A^{-1}>0,\left(\beta_{i i} \cdot D_{i i}\right)^{-1}>0$ for $i \in\lceil i, n]$ and $\{1\}$ is deleted from the ranges of the entries of $\beta_{i j}$ for $(i, j) \in V$ and of $\overline{\beta_{i j}}$, then $\rho(T)$ is

(1) a decreasing function of $\beta_{i j}$ for $(i, j) \in V$;

(2) an increasing function of $\beta_{i i}^{+}$;

(3) a decreasing function of $\overline{B_{i i}}$ when $b_{k}^{i}$ is kept constant;

(4) an increasing function of $\theta_{i}$ when all the entries of $\beta_{i i}$ are set equal to $\theta_{i}$

(5) a decreasing function of $W$. 


\section{$\underline{\text { Proof }}$}

Let

$$
A=M-N
$$

be the splitting associated with the block Oliphant-Buleev-Woznicki method; we first show that if $\mathrm{P}$ is monotone then

$$
\mathrm{AD}_{\beta}^{-1}=\mathrm{MD}_{\beta}^{-1}-\mathrm{ND}_{\beta}^{-1}
$$

is a regular splitting of $\mathrm{AD}_{\beta}^{-1}$.

We have indeed

$$
\begin{aligned}
& D_{B} M^{-1}=\left(\sum_{k=0}^{n-1}\left(D_{B} P^{-1}\left(F_{B} D_{B}^{-1}+H D_{B}^{-1}\right)\right)^{k}\right)\left(\sum_{\ell=0}^{n-1}\left(D_{B} P^{-1}\left(E_{B} D_{B}^{-1}+G D_{B}^{-1}\right)\right)^{\ell}\right) D_{B} P^{-1} \\
& \mathrm{GD}_{\beta}^{-1}+\mathrm{HD}_{\beta}^{-1}=\operatorname{rest}_{W}\left(\left(\mathrm{E}_{\beta} \mathrm{D}_{\beta}^{-1}+\mathrm{GD}_{\beta}^{-1}\right) \mathrm{D}_{\beta} \mathrm{P}^{-1}\left(\mathrm{~F}_{\beta} \mathrm{D}_{\beta}^{-1}+\mathrm{HD}_{\beta}^{-1}\right)\right) \\
& P \leq D_{\beta}
\end{aligned}
$$

and, since $\mathrm{D}_{\beta}^{-1} \geq 0$

$$
E_{\beta} D_{\beta}^{-1} \geq 0 \quad F_{B} D_{\beta}^{-1} \geq 0
$$

Therefore, if $\mathrm{P}$ is monotone,

$$
D_{\beta} \mathrm{P}^{-1} \geq 0
$$

and then, by the preceding relations

$$
D_{B} M^{-1} \geq 0
$$

On the other hand

$$
\begin{aligned}
& \mathrm{ND}_{\beta}^{-1}=\left(I-\mathrm{DD}_{\beta}^{-1}\right)+\left(\mathrm{E}-\mathrm{E}_{\beta}\right) \mathrm{D}_{\beta}^{-1}+\left(\mathrm{F}-\mathrm{F}_{B}\right) \mathrm{D}_{\beta}^{-1} \\
& \quad+\left(\text { off block diag }\left(\left(\mathrm{E}_{\beta} \mathrm{D}_{\beta}^{-1}+\mathrm{GD}_{\beta}^{-1}\right) \mathrm{D}_{\beta} \mathrm{P}^{-1}\left(\mathrm{~F}_{\beta} \mathrm{D}_{\beta}^{-1}+\mathrm{HD}_{\beta}^{-1}\right)\right)-\left(\mathrm{GD}_{\beta}^{-1}+\mathrm{HD}_{B}^{-1}\right)\right)
\end{aligned}
$$

and, letting $B$ be the purely diagonal matrix whose entry on line $k$ of block (i, i) is $b_{k}^{i}$, we have

$$
D_{\beta} \geq B D
$$

thus 


$$
\begin{aligned}
& I \geq B^{-1} \geq D_{\beta}^{-1} \\
& I-D_{\beta}^{-1} \geq 0
\end{aligned}
$$

Therefore

$$
\mathrm{ND}_{\beta}^{-1} \geq 0
$$

As a consequence,

$$
\mathrm{NM}^{-1}=\mathrm{ND}_{\beta}^{-1} \mathrm{D}_{\beta} \mathrm{M}^{-1} \geq 0
$$

and the splitting (247) is a weak regular splitting.

Therefore, if $\mathrm{P}$ is monotone and $\rho\left(\mathrm{M}^{-1} \mathrm{~N}\right)<1$, then $\mathrm{A}$ is monotone by theorem 1. On the other hand, if $A$ is monotone, there exists $x \geq 0$ such that $\mathrm{Ax}>0$ by theorem 17 ; since

$$
A \leq D
$$

and, by (256)

$$
\mathrm{D}_{B}^{-1} \mathrm{BD} \leq \mathrm{I}
$$

We have also

$$
D_{R}^{-1} B A \leq D_{B}^{-1} B D \leq I
$$

thus $\mathrm{A}$ is a block-M-matrix by theorem 17 .

Conversely, if $\mathrm{A}$ is a block-M-matrix, then

$$
A=D-(E+F)
$$

is a regular splitting and, by theorem 1 ,

$$
\rho\left(D^{-1}(E+F)\right)<1
$$

But

$$
A_{B}=D_{B}-\left(E_{B}+F_{B}\right)
$$

is also a regular splitting with

$$
\begin{gathered}
1 \quad E_{\beta}+F_{\beta} \leq E+F \\
\text { and, by (256) since } D^{-1} \geq 0, \\
D_{\beta}^{-1} \leq D^{-1}
\end{gathered}
$$

Therefore

$$
\rho\left(D_{\beta}^{-1}\left(E_{\beta}+F_{\beta}\right)\right) \leq \rho\left(D^{-1}(E+F)\right)<1
$$


and $A_{B}$ is monotone (thus a block-M-matrix) by theorem 1 . Therefore $P$ is monotone by lemma 5 and $\rho\left(M^{-1} N\right)<1$ by theorem 1 .

For the last parts of the proof, we observe that, if $\mathrm{A}$ is a block-M-matrix, then $A_{B}$ is also a block-M-matrix as observed above; thus

$$
A_{B} D^{-1}
$$

is a block-M-matrix and

$$
\mathrm{PD}^{-1}
$$

is monotone by lemma 5 ; then it is seen that

$$
\mathrm{AD}^{-1}=\mathrm{MD}^{-1}-\mathrm{ND}^{-1}
$$

is a regular splitting of $\mathrm{AD}^{-1}$.

The proof is concluded as that of theorem 13 .

\section{Remarks}

1. As in the case of theorem 13, the ranges of the entries of $\beta_{i i}$ may also be defined by using the columns of $\beta_{i i}$ in place of the lines. 2. If all the entries of $\beta_{i i}$ are equal to one and the same number $\theta_{i} \geq 1$, then clearly the condition $\left(\beta_{i i} \cdot D_{i i}\right)^{-1} \geq 0($ or $>0)$ is equivalent to $D_{i i}^{-1} \geq 0($ or $>0)$. 


\subsection{The characterization of block-M-matrices}

The following results will be stated without proofs whenever

these are similar to the proofs of the corresponding results of M-matrices.

Lemma 6

Let $A=K-L-U$ where $K^{-1} \geq 0, L \geq 0$ and $U \geq 0$ are respectively block-diagonal, strictly lower and upper block-triangular matrices and let the block-diagonal, strictly lower and upper block-triangular matrices $P, G$ and $H$ respectively be defined by

$$
\begin{aligned}
& P=K-\operatorname{block-diag}\left((L+G) P^{-1}(U+H)\right) \\
& G+H=\operatorname{off} \text { block-diag }\left((L+G) P^{-1}(U+H)\right)
\end{aligned}
$$

Then $P$ is monotone if and only if $A$ is a block-M-matrix.

Theorem 19

Let $A=K-L-U$ where $K^{-1} \geq 0, L \geq 0$ and $U \geq 0$ are respectively block-diagonal, strictly lower and upper block-triangular matrices and let $D_{i}, i=1,2 \ldots$. $n$, be the leading principal minors of A relative to the same partitioning; let the block-diagonal, strictly lower and upper block-triangular matrices $P, G$ and $H$ respectively be defined by

$$
\begin{aligned}
& P=K-\operatorname{block-diag}\left((I+G) P^{-1}(U+H)\right) \\
& G+H=\operatorname{rest}_{W}\left((L+G) P^{-1}(U+H)\right)
\end{aligned}
$$

where $W$ is an arbitrary set of off-diagonal indices.

If $\mathrm{A}$ is a block-M-matrix, $\mathrm{P}$ is monotone and

$$
D_{i} \leq \prod_{s=1}^{i} \operatorname{det}\left(P_{s s}\right)
$$

with equality if $W$ contains the block-Choleski set of $A$, for the same partitioning. 


\section{Theorem 20}

Let $A=K-L-U$ where $K^{-1} \geq 0, L \geq 0$ and $U \geq 0$ are respectively block-diagonal, strictly lower and upper block-triangular matrices.

Then the following conditions are equivalent to each other:

$1 \%$ there exists a vector $\mathrm{x} \geq 0$ such that $\mathrm{Ax}>0$;

$2 \%$ there exists a vector $\mathrm{x}>0$ such that $\mathrm{Ax}>0$;

$3^{0} \%$ the inverse $A^{-1}$ of $A$ exists and $A^{-1} \geq 0$

$4 \%$ the matrix A may be written in the form $R S$ where $R$ and $S$ are respectively lower and upper block-triangular block-M-matrices according to the given partitioning;

$5^{\circ} /$ the matrix $\mathrm{K}^{-1} \mathrm{~A}$ is an M-matrix.

$\underline{\text { Remark }}$

From theorem $20,5^{\circ}$, it is seen that all the criteria set up by Fiedler and Pták [3] for M-matrices can possibly be used for identifying block-M-matrices .

\subsection{Criteria for arbitrary complex matrices}

On the basis of theorem $20,5^{\circ}$, we have the following extension of lemma 4 to the case of block-M-matrices:

\section{Lemma 7}

Let $A=K-L-U$ be the decomposition of the arbitrary $n \times n$ partitioned complex matrix A into its block-diagonal, strictly lower and upper blocktriangular parts; let the block-diagonal, strictly lower and upper blocktriangular matrices $\mathrm{P}, \mathrm{G}$ and $\mathrm{H}$ respectively be defined by

$$
\begin{aligned}
& P=K-\text { blockdiag }\left((L+G) P^{-1}(U+H)\right) \\
& G+H=\operatorname{rest}_{W}\left((L+G) P^{-1}(U+H)\right)
\end{aligned}
$$

where $W$ is an arbitrary set of off-block-diagonal entries; let $K$ be non singular and

$$
P\left(K^{-1} A\right)=I-L_{1}-U_{1}
$$

be the decomposition of $\mathrm{P}\left(\mathrm{K}^{-1} \mathrm{~A}\right)$ into its block-diagonal, strictly 
lower and upper block-triangular parts, according to the same partitioning; let $\mathrm{P}_{1}, \mathrm{G}_{1}$ and $\mathrm{H}_{1}$ be defined by (278) and (279) applied to $I, L_{1}$ and $U_{1}$.

If $\mathrm{P}\left(\mathrm{K}^{-1} \mathrm{~A}\right)$ is an M-matrix, then $\mathrm{P}$ is non-singular; we have moreover

$$
\left|P^{-1} K\right| \leq P_{1}^{-1} \quad\left|K^{-1} G\right| \leq G_{1} \quad\left|K^{-1} H\right| \leq H_{1}
$$

$\underline{\text { Proof }}$

From Eq. (278) and (279), it is seen that

$$
\begin{aligned}
& \tilde{P}=I-\operatorname{block}-\operatorname{diag}\left((\tilde{L}+\tilde{G}) \tilde{P}^{-1}(\tilde{U}+\tilde{H})\right) \\
& \left.\tilde{G}+\tilde{H}=\operatorname{rest}_{W}(\tilde{L}+\tilde{G}) \tilde{P}^{-1}(\tilde{U}+\tilde{H})\right)
\end{aligned}
$$

where

$$
\begin{aligned}
& \tilde{\mathrm{L}}=\mathrm{K}^{-1} \mathrm{~L} \quad \tilde{\mathrm{U}}=\mathrm{K}^{-1} \mathrm{U} \\
& \tilde{\mathrm{P}}=\mathrm{K}^{-1} \mathrm{P} \quad \tilde{\mathrm{G}}=\mathrm{K}^{-1} \mathrm{G} \quad \tilde{\mathrm{H}}=\mathrm{K}^{-1} \mathrm{H}
\end{aligned}
$$

Since we have

$$
\mathrm{L}_{1}=|\tilde{\mathrm{L}}| \quad \mathrm{U}_{1}=|\tilde{\mathrm{U}}|
$$

the proposition follows from lemma 4.

Corollary

Let $A$ be an arbitrary partitioned complex matrix with non-singular block-diagonal $\mathrm{K}$; if $\mathrm{P}\left(\mathrm{K}^{-1} \mathrm{~A}\right)$ is an M-matrix, then $\mathrm{A}$ is non-singular and

$$
\left|A^{-1} K\right| \leq\left(P\left(K^{-1} A\right)\right)^{-1}
$$

\section{Theorem 21}

Let $\mathrm{A}$ be an arbitrary $\mathrm{n} x \mathrm{n}$ partitioned complex matrix with non-singular block-diagonal $D$; if $P\left(D^{-1} A\right)$ is an M-matrix, if the entries of each block $\beta_{i j}$ are al1 equal to one and the same number $\theta_{i j}$ with $0 \leq \theta_{i j} \leq 1$ for $(i, j) \in V$ and $\theta_{i j} \geq 1$ for $i \in[1, n]$, then the block-diagonal matrix $P$ of the block Oliphant-Buleev-Woznicki method is non-singular and the method is convergent. Moreover its convergence rate is not less than the convergence rate 
of the same method applied to $\mathrm{P}\left(\mathrm{D}^{-1} \mathrm{~A}\right)$.

\section{$\underline{\text { Proof }}$}

Let $A=M-N$ be the corresponding splitting; let

$$
P\left(D^{-1} A\right)=M_{1}-N_{1}
$$

be the splitting corresponding to the same method, applied to $P\left(D^{-1} A\right)$.

As in the proof of theorem 8, we have, by lemma 7 ,

$$
\left|M^{-1} D\right| \leq M_{1}^{-1}
$$

and

$$
\left|\mathrm{D}^{-1} \mathrm{~N}\right| \leq \mathrm{N}_{1}
$$

Therefore

$$
\left|M^{-1} N\right|=\left|M^{-1} D D^{-1} N\right| \leq\left|M^{-1} D\right|\left|D^{-1} N\right| \leq M_{1}^{-1} N_{1}
$$

and, by theorem 7 ,

$$
\rho(\mathrm{T}) \leq \rho\left(\mathrm{T}_{1}\right)
$$

where $\mathrm{T}=\mathrm{M}^{-1} \mathrm{~N}$ and $\mathrm{T}_{1}=\mathrm{M}_{1}^{-1} \mathrm{~N}_{1}$.

The conclusion follows from theorem 13. 
7 Concluding remarks

In this report we have obtained factorizability conditions and convergence criteria of point and block factorization iterative methods for general matrices.

These developments have been obtained by theoretical extensions of our previous results for essentially non-negative matrices [1] and they give, as auxiliary results, some new forms of characterization properties of M-matrices obtained by Fiedler and Ptak [3].

For general matrices, these developments lead to consider criteria which insure that a given essentially non-negative matrix is an M-matrix. In this respect, we hope that the sufficient conditions set up by Fiedler and Ptak will open the field to applications. 
References

[1] R. Beauwens, "Convergence analysis of some factorization iterative me thods for M-matrices", Series in Applied Mathematics, Report No. 73-7 Northwestern University (1973).

[2] R. Beauvens and $M$. Borysiewicz, "On the convergence of block-iterative and factorization iterative methods for symnetric or unsymmetric complex matrices", Internal Report, Universite Libre de Brurelles (1973).

[3] M. Fiedler and V. Pták, "On matrices with non-positive off-diagonal elements and positive principal minors", Czech. Math. Journal, 12, $382-400$ (1962).

[4] T. Dupont, R. P. Kendall and H. H. Rachford, "An approximate factorization procedure for solving self-adjoint elliptic difference equations", SIAM J. Numer. Ana1., 5, 559-573 (1968).

[5] N. T. Buleev, "A numerical method for the solution of two-dimensional and three-dimensional equations of diffusion", Mat. Sb., 51, 227-238 (1960) Russia.

[6] R. Beauwens, "An order relation between factorization iterative methods", Internal Report, Universite Libre de Bruxelles (1973).

[7] R. S. Varga, "Matrix iterative analysis", Prentice Hal1 (1962).

[8] J. M. Ortega and W. C. Rheinboldt, "Monotone iterations for nonlinear equations with application to Gauss Numer. Ana1., $4,171-190$ (1967).

[9] H. S. Price, "Monotone and ascillation matrices applied to finite difference approximations", Math.Comp., 22, 489-516 (1968).

[10] J. S. Vandergraft, "Application of partial orderings to the study of positive definiteness, monotonicity and convergence of iterative methods for linear systems", SIAM J. Numer. Ana1., 9, 97-104. (1972).

[11] Z. Woznicki, "Two-sweep iterative methods for solving large linear systems and their application to the numerical solution of multi-group multi-dimensional neutron diffusion equation", Doctoral Dissertation, Institute of Nuclear Research, Swierk (1973).

[12] Ky Fan, "Topological proof for certain theorems on matrices with nonnegative elements", Monatsh. Math., 62, 219-237 (1958).

[13] N. Dunford and J. T. Schwartz, "Linear Operators", Part I, Interscience (1964).

[14] R. S. Varga, "Factorization and normalized iterative methods", Boundary Problems in Differential Equations, edited by R. E. Langer, University of. Wisconsin Press, Madison, 121-142 (1960).

[15] G. P. H. Styan, "Hadamard Products and Multivariate Statistical Analysis" Lin. Alg. and App1., 6, 217-240 (1973). 
- 2 iormatcist

AECM 3201

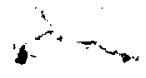

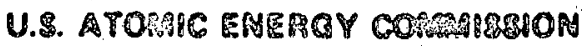

UNIVERSITY-TYPE CONTAACTOA'S RECOMMENDATION FOR DISPOSITION OF SCIENTIFIC AHD TECHAICAL DOCUMEAT

(Sto instructions on Rowere Sinte)

1. AEC REPORT NO.

COO-2280-7

2. TITLE

ON THE POINT AND BLOCK FACTORIZATION ITERATIVE

METHODS FOR ABBITRARY MATRICES AND THE CHARACTERIZATION OF.

3. TYPE OF OOCUMENT (ChOCK ONO):

Q. Scientific and technicel report

b. Contornence pspar

Tille of conference

Dete of conforence

Exact locstion of conforence.

Sponserind orgenization

c. Jouraal article. "Submittéd to

a. Oiner (spocify)

4. RE COMAMENDED ANNOUNCEAENT ANO DISTRIEUTIONA

(Chock ons):

8 a. AEC's normal announcomoni and distributuon procedures mey be pollawed.

b. Make avaitade only within AEC end 10 AEC coneractors ond other U.S. Government ajencies and inoir contractors.

3. REASON FOA AECOMAEADED RESTRICTIONB:

6. SUBAITTED BY: NAME AND POSITION (PIEAse print or typo)

E. H. Bareiss

Professor and Principal Investigator

Organization

Department of Computer Sciences and Engineering Sciences

Northwestern University

Evanston, I11inois 60201

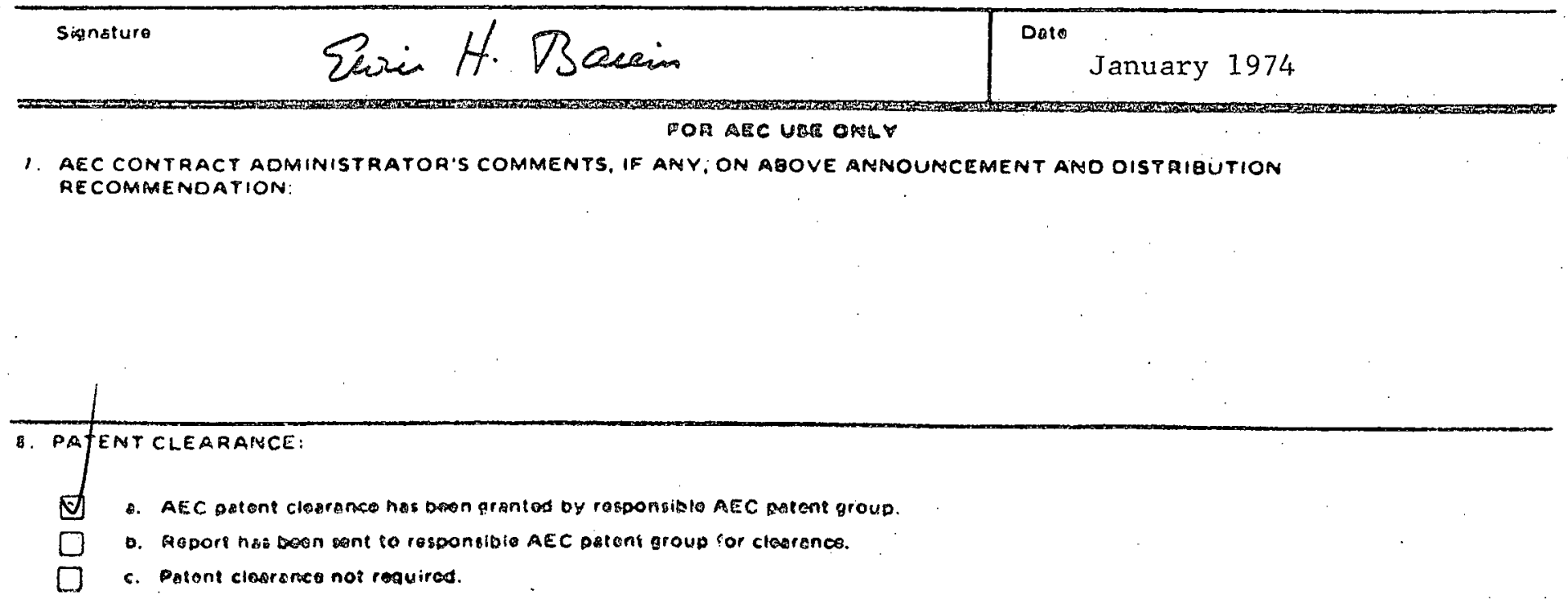

